\title{
Astrobiological Stoichiometry
}

\author{
Patrick A. Young, Steven J. Desch, Ariel D. Anbar, ${ }^{1,2}$ Rory Barnes, ${ }^{3}$ Natalie R. Hinkel, ${ }^{4}$ \\ Ravikumar Kopparapu, ${ }^{5}$ Nikku Madhusudhan, ${ }^{6}$ Nikhil Monga, Michael D. Pagano, \\ Miriam A. Riner, ${ }^{7}$ Evan Scannapieco, Sang-Heon Shim, and Amanda Truitt ${ }^{1}$
}

\begin{abstract}
Chemical composition affects virtually all aspects of astrobiology, from stellar astrophysics to molecular biology. We present a synopsis of the research results presented at the "Stellar Stoichiometry" Workshop Without Walls hosted at Arizona State University April 11-12, 2013, under the auspices of the NASA Astrobiology Institute. The results focus on the measurement of chemical abundances and the effects of composition on processes from stellar to planetary scales. Of particular interest were the scientific connections between processes in these normally disparate fields. Measuring the abundances of elements in stars and giant and terrestrial planets poses substantial difficulties in technique and interpretation. One of the motivations for this conference was the fact that determinations of the abundance of a given element in a single star by different groups can differ by more than their quoted errors. The problems affecting the reliability of abundance estimations and their inherent limitations are discussed. When these problems are taken into consideration, self-consistent surveys of stellar abundances show that there is still substantial variation (factors of $\sim 2$ ) in the ratios of common elements (e.g., $\mathrm{C}, \mathrm{O}, \mathrm{Na}, \mathrm{Al}, \mathrm{Mg}, \mathrm{Si}$, $\mathrm{Ca}$ important in rock-forming minerals, atmospheres, and biology. We consider how abundance variations arise through injection of supernova nucleosynthesis products into star-forming material and through photoevaporation of protoplanetary disks. The effects of composition on stellar evolution are substantial, and coupled with planetary atmosphere models can result in predicted habitable zone extents that vary by many tens of percent. Variations in the bulk composition of planets can affect rates of radiogenic heating and substantially change the mineralogy of planetary interiors, affecting properties such as convection and energy transport. Key Words: SupernovaeChemical evolution-Extrasolar planets-Geochemistry—Habitability. Astrobiology 14, 603-626.
\end{abstract}

\section{Introduction}

A SK AN ASTRONOMER to draw the periodic table of the elements, and it may look something like Fig. 1. Stars are almost entirely hydrogen and helium. Using the abundances of Lodders (2010) for the Sun's initial composition, $\mathrm{H}$ and He make up $\sim 98.5 \%$ of the mass. The species $\mathrm{O}, \mathrm{C}$, $\mathrm{Fe}, \mathrm{Ne}, \mathrm{Si}, \mathrm{N}, \mathrm{Mg}$, and $\mathrm{S}$ (in that order) make up $\sim 1.5 \%$ of the mass. However, these elements are central in the thoughts of planetary scientists, geochemists, geophysicists, and astrobiologists as they try to understand how planets form and function and how they evolve as abodes for life. Understanding their origin and distribution are therefore fundamental to un- derstanding the potential habitability of extrasolar planetary systems.

Whereas $\mathrm{H}$ and $\mathrm{He}$ are primordial, created in the Big Bang, other elements, referred to as "metals" by astronomers, largely are not. Following the Big Bang, Li, Be, and B represented one-billionth of the mass, and heavier elements were present at a level of one part in $10^{12}$ (Steigman, 2012). Virtually all the metals present today were created by nucleosynthesis in a variety of stellar environments.

Because $\mathrm{Fe}$ is abundant and has many atomic transitions easily observed via optical absorption in a stellar atmosphere, astronomers measure Fe abundance as an indicator of the chemical composition of a star. The Fe/H abundance

\footnotetext{
${ }^{1}$ School of Earth and Space Exploration, Arizona State University, Tempe, Arizona.

${ }^{2}$ Department of Chemistry and Biochemistry, Arizona State University, Tempe, Arizona.

${ }^{3}$ Department of Astronomy, University of Washington, Seattle, Washington.

${ }^{4}$ Department of Physics and Astronomy, San Francisco State University, San Francisco, California.

${ }^{5}$ Virtual Planetary Laboratory, Center for Exoplanets and Habitable Worlds, Penn State Astrobiology Research Center, Department of Geosciences, Pennsylvania State University, University Park, Pennsylvania.

${ }^{6}$ Department of Astronomy, Yale University, New Haven, Connecticut.

${ }^{7}$ Planetary Science Institute, Tucson, Arizona.
} 


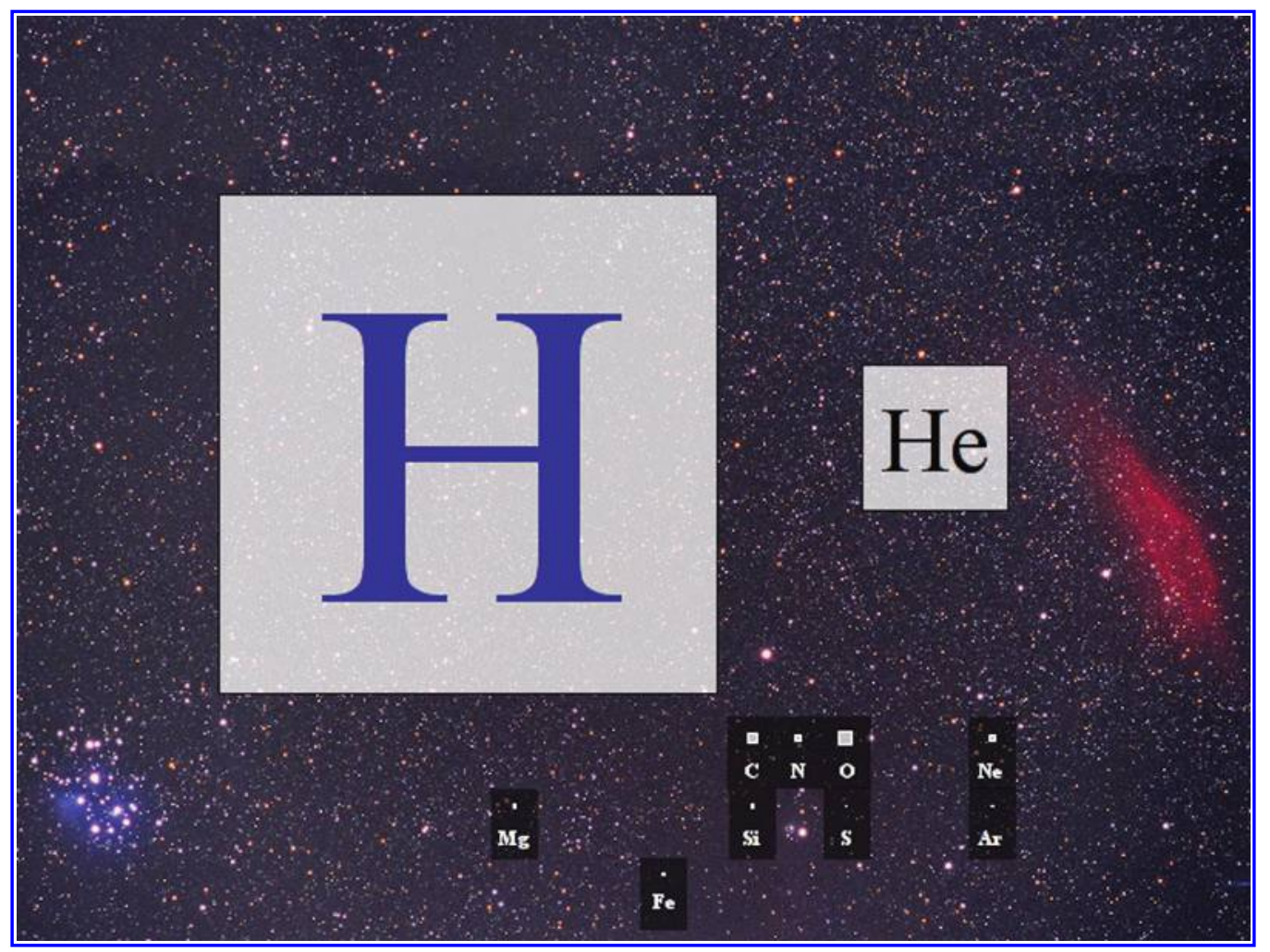

FIG. 1. An astronomer's periodic table of the elements. The size of each element's white square measures the abundance of that element (by number) in the Sun. Elements heavier than He are termed "metals." Because the Sun is considered to sample the average interstellar medium, it is presumed that all stars will share the Sun's relative proportions of metals. The relative sizes of the boxes for $\mathrm{C}$, $\mathrm{O}$, etc., are presumed to be the same for all stars. One of the surprises of stellar spectroscopy studies is that this presumption is false. The observed variations in stellar abundance ratios have significant consequences for star and planet formation evolution and for habitability. (From http://bjm.scs.illinois.edu/astrochem.php) Color images available online at www.liebertonline.com/ast

ratio in a star is usually reported as the logarithm of the number ratio of these elements scaled to the ratio in the Sun, denoted $[\mathrm{Fe} / \mathrm{H}]$ (Helfer et al., 1959). In other words, $[\mathrm{Fe} / \mathrm{H}]=$ $\log _{10}\left[(\mathrm{Fe} / \mathrm{H})_{\text {star }} /(\mathrm{Fe} / \mathrm{H})_{\text {Sun }}\right]$.

We can discuss other elemental ratios normalized to the Sun in the same way. It is common to use the same notation to quantify elemental ratios relative to $\mathrm{Fe}$, since $\mathrm{Fe}$ is most commonly measured. For example, $[\mathrm{O} / \mathrm{Fe}]=\log _{10}[(\mathrm{O} /$ $\left.\mathrm{Fe})_{\text {star }} /(\mathrm{O} / \mathrm{Fe})_{\text {Sun }}\right]$.

The term "metallicity" is commonly used by astronomers. Formally, it refers to the total amount of all heavy elements in the star. Practically, however, metallicity and $[\mathrm{Fe} / \mathrm{H}]$ are often used interchangeably because thousands of stars have measured $[\mathrm{Fe} / \mathrm{H}]$, but many fewer have complete measurements for even the most abundant subset of elements. Using metallicity and $\mathrm{Fe}$ content in this way implicitly makes the assumption that all metals scale with $\mathrm{Fe}$ in the same proportions we observe in the Sun, as if the relative sizes of the boxes in the astronomer's periodic table looked exactly the same as Fig. 1, for every star. In this article we will use metallicity to refer to $[\mathrm{Fe} / \mathrm{H}]$ only. For example, when we say a star has one-third the metallicity of the Sun, $Z=1 / 3 Z_{\text {Sun }}$, we mean only $[\mathrm{Fe} / \mathrm{H}]=-0.5$.

One of the lessons of stellar spectroscopy over the last decade or so is that the elemental abundances of stars do not all scale directly with the metallicity. Data exhibiting variation in abundance ratios has existed for some time, but much improved statistics and recognition of the potential signifi- cance of this variation were substantially driven by the advent of large-scale efforts at planet detection and characterization. At the same time, a bonanza of exoplanet discoveries has revealed an incredible diversity of planet types of which our solar system represents only a small subsample. The range in elemental abundances and the diversity of planet types are probably not unrelated; these differences can have significant consequences for the evolution of stars, the formation of planets, the processes that occur within planets, and ultimately the habitability of a stellar system.

Following the thread of elements-from astronomical scales to planetary settings to biological ecosystems-has been a major thrust of the Arizona State University "Follow the Elements" team of the NASA Astrobiology Institute (NAI). These themes are sure to figure prominently in future astrobiology research as well. With these thoughts in mind, on April 11-12, 2013, Arizona State University hosted an NAI Workshop Without Walls on the theme of elemental abundance ratios, entitled "Stellar Stoichiometry." The Web site for the conference http://stellarstoich.sese.asu.edu (or https://sites.google.com/a/asu.edu/stellarstoich) describes the conference and archives electronic versions of the presentations. A companion article (Desch et al., 2014) describes the conference itself. This article represents a synopsis of the scientific presentations from that workshop.

The themes explored in the workshop and in this article are as follows (as this is in part a conference report, the 
primary contributors are identified for each section): In Section 2, we discuss the process and results of observationally determining the abundances of stars and planets. Inferring stellar abundances is a challenge, but the data show that elemental abundance ratios do vary significantly from star to star. A link between stellar abundances and planet formation is suggested. We have tantalizing direct measurements of abundances in exoplanets, but the interpretation of those preliminary measurements is far from clear. We also present cautionary tales of attempts to measure abundances of planets in our own solar system. In Section 3, we examine the consequences of elemental abundance variations for aspects of habitability. We consider stellar evolution models and planetary climate models and their effects on the time evolution of habitable zones (HZs). We also consider the consequences for heating and structure and dynamics within planets. In Section 4, we discuss factors that can lead to variable abundance ratios in stars, such as injection of supernova material, and in planets, such as volatile transport in protoplanetary disks. We summarize these diverse findings in Section 5, where we conclude that known elemental abundance ratio variations will have significant consequences for star and planet formation and evolution and for the habitability of a stellar system.

\section{Finding Abundances of Stars and Planets}

The first step in assessing the effect of abundances on any aspect of habitability is to determine the actual abundances of the stars and planets. This is by no means a trivial exercise, even for the stars, which are much brighter and easier to observe. The high-resolution spectra that are needed are burdensome to obtain, and for many nearby stars only the abundances of Fe, or at most a few elements, are determined. The measurement is fraught with uncertainty, often from unrecognized sources. Nevertheless, self-consistent surveys reveal that there are substantial intrinsic variations in elemental abundance ratios, even between stars of similar metallicity.

As difficult as it is to infer stellar abundances, observationally constraining planetary abundances is even more difficult, although it is becoming increasingly tractable. Absorption spectra of gas giant planet atmospheres allow some constraints on elemental abundances, but inferences about bulk abundances in both terrestrial and gas giant planets remain difficult even in the planets of our own solar system.

\subsection{Stellar abundance measurements (Natalie Hinkel)}

Hinkel (2012) compiled spectroscopic abundance data for 50 elements across 3069 stars within 150 pc of the Sun from 84 literature sources to produce the Hypatia Catalog. The goal of Hypatia is to examine nearby stars and regions in the solar neighborhood for chemical abundance trends.

When analyzing the abundance measurements taken across 25 years from different groups at a variety of instruments, there is potential for many variations between the data sets. While some effort has been made to compare new abundance determinations with more standard benchmark catalogs, for example, Edvardsson et al. (1993), Thévenin and Idiart (1999), or Valenti and Fischer (2005), those comparisons are not standardized, varying in many ways from the statistical to graphical. The unfortunate result is that different groups often measure significantly different abundances for the same element in the same star. Figure 2, from the work of Hinkel (2012), illustrates how different research groups measured the elements $\mathrm{Na}, \mathrm{Si}, \mathrm{O}, \mathrm{Sc}, \mathrm{Al}$, and $\mathrm{Fe}$ in five different stars (the data sets are discussed and the research groups identified by Hinkel, 2012). The maximum abundance measurement minus the minimum for each element within a star is referred to as the "spread." The observational spread in $[\mathrm{Na} / \mathrm{H}]$ within $\mathrm{HIP} 79672$, for example, is $\sim 0.1$ dex, which greatly exceeds the quoted observational errors for many groups, $\sim 0.04$ dex. Different groups must be measuring stellar abundances using inconsistent methodologies, since the variation in abundance determinations goes far beyond observational errors.

One complication is that different groups scale to different assumed solar abundances such as those of Holweger (1979), Anders and Grevesse (1989), and Asplund et al. (2009), although ultimately this is not the main source of the discrepancy. The differences between solar abundance determinations are dishearteningly large. The quoted abundances for the primordial solar composition are derived from a mixture of techniques, primarily analysis of primitive meteorites and spectroscopy of the solar photosphere (Lodders, 2010). Photospheric abundances face two problems. First, the measured abundances must be corrected for stellar processes such as gravitational settling of heavy elements over the life of the Sun by using stellar models. The second and larger problem is that the translation of measured widths of spectral lines to an actual number of atoms requires a stellar atmosphere model. The 3-D models of the solar photosphere and convection zone that have been developed over the last decade give substantially different abundances for some species than the old 1-D approximations (Asplund et al., 2009). One of the most strongly affected species is oxygen, the third most abundant element in the Universe.

All the abundances within the Hypatia Catalog were corrected for the solar normalizations applied by each individual group and then renormalized according to the same scale, namely, Lodders (2010) as described by Hinkel and Kane (2013). For example, Valenti and Fischer (2005) determined that HIP 490 has $[\mathrm{Ti} / \mathrm{H}]=0.05$, using the solar abundance scale of Anders and Grevesse (1989) for which $\log _{10} \varepsilon(\mathrm{Ti})=4.99$. (Since solar abundances are absolute, we cannot use the $[X / H]$ notation. In this notation, $\log _{10} \varepsilon(X)$ is the total number of atoms of $X$ normalized to $\log _{10} \varepsilon(\mathrm{H})=$ 12.00). For comparison, Lodders (2010) gave $\log _{10} \varepsilon(\mathrm{Ti})=$ 4.93. Renormalizing the Valenti and Fischer (2005) result $[\mathrm{Ti} / \mathrm{H}]=0.05$ to the Lodders (2010) abundance scale, we find $[\mathrm{Ti} / \mathrm{H}]=0.05+4.99-4.93=0.11 \mathrm{dex}$. When this method was applied to all the elements within the Hypatia stars, the median absolute difference between the original and renormalized abundances was 0.04 dex. The change resulting from renormalization was often larger than the reported error, but the total effect of applying a consistent solar renormalization on the abundance spread was minimal, actually increasing it from 0.14 to 0.15 dex.

It is clear from the analysis performed on the Hypatia Catalog that not only are there some major discrepancies between different stellar abundance data sets for the same elements in the same stars but the remedy must be more than cosmetic. An evaluation of the stellar parameters, such as effective temperature and surface gravity, reported by multiple authors indicates that stellar atmosphere models 


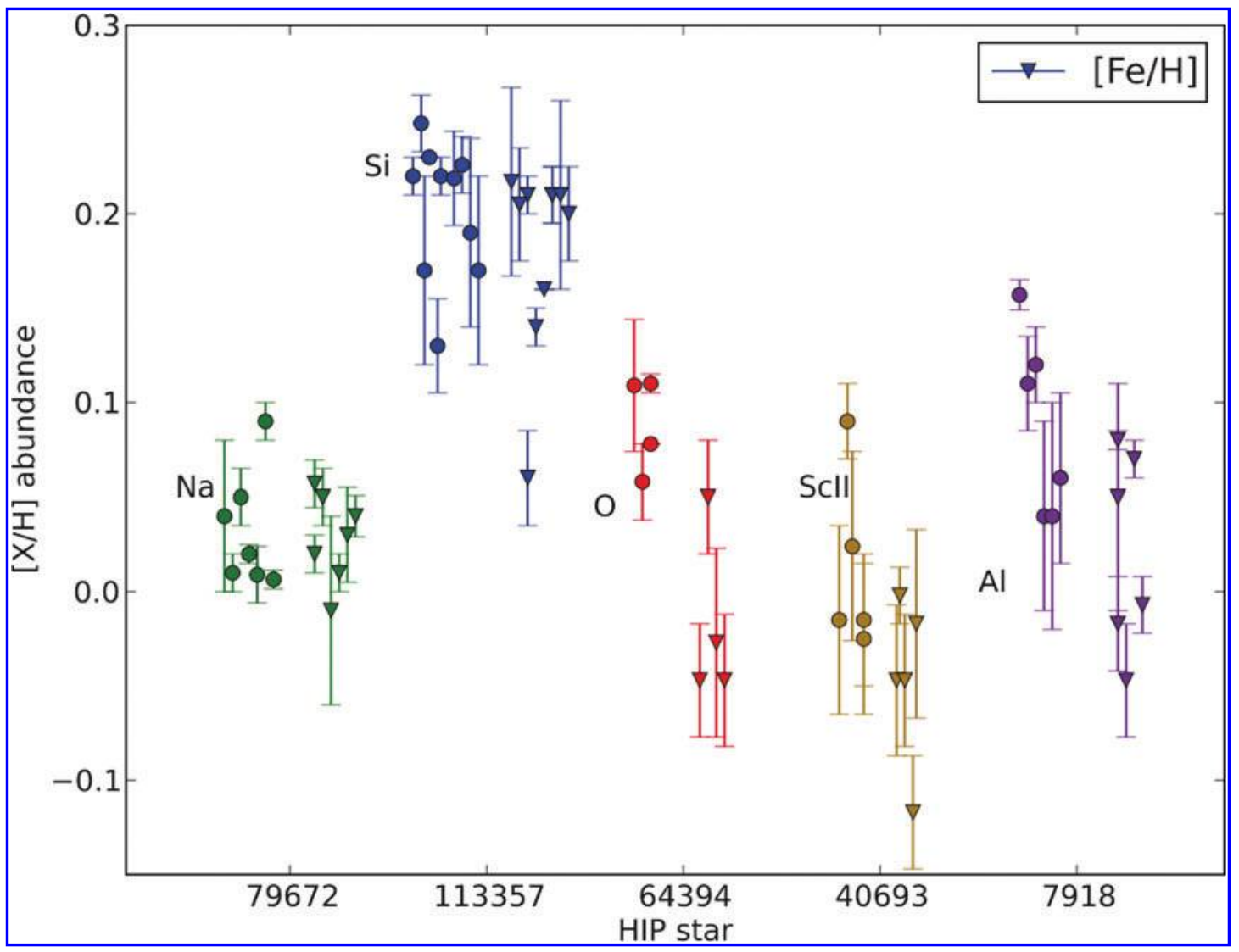

FIG. 2. Abundances of various elements in five stars, as measured by different groups, taken from various catalogs (Hinkel, 2012). For HIP 79672, measurements of Na (circles) and Fe (triangles) are displayed. For HIP 113357, measurements of Si (circles) and Fe (triangles) are displayed, etc. Error bars denote uncertainties quoted in the cited catalogs. The cumulative spread (maximum abundance determination minus the minimum) is larger than the typical quoted errors of the individual measurements. Color images available online at www.liebertonline.com/ast

might be the source of the problem. However, the varying number and quality of spectral lines might also cause the large amount of spread. Even atomic data such as oscillator strength can be shown to cause substantial variations. Many of these factors are examined systematically in a companion paper arising from the workshop and in preparation for the Astrophysical Journal (Hinkel et al., unpublished). This work is receiving contributions from five independent groups applying their methods to the same set of observational data. Fixed values for stellar parameters, atomic data, equivalent width measurements of spectral line strength, and line lists are provided so that each factor may be independently varied and the source of discrepancies identified between methods. It is hoped that this will result in a recommended set of best practices.

The preliminary results for four stars and three groups presented at the workshop itself indicate that line lists do have a substantial effect. Differences in temperature $T$, surface gravity $\log (g)$, and $[\mathrm{Fe} / \mathrm{H}]$ were up to $266 \mathrm{~K}$, $0.05 \mathrm{dex}$, and $0.36 \mathrm{dex}$, respectively. Imposing fixed stellar parameters typically changed $\Delta[\mathrm{Fe} / \mathrm{H}]$ between groups by $0-0.08$ dex. Most other elements exhibited the same behavior. Large disparities exist for elements that have either one weak line that is difficult to measure or have many lines of which a subset are chosen for doing the analysis. In at least one case a difference in the oscillator strength used for calculation of electron transitions in $\mathrm{Mg}$ produced a significant disparity.

\subsection{Variations in stellar abundance ratios of nearby stars (Michael Pagano)}

To judge the physical effects of elemental abundance ratios, we must find statistical methods to separate the observational and methodological sources of scatter from the physical variation in abundance ratios exhibited by stars, which we refer to as the "intrinsic scatter." Disentangling the intrinsic scatter from the actual observational errors is not straightforward. To do so, we performed a linear regression ( $\chi^{2}$ fit) (Isobe et al., 1990) in which the total scatter is assumed to be the sum in quadrature of an observational uncertainty $u_{\text {obs }}$ and an intrinsic scatter $u_{\text {intrinsic }}$. The goodnessof-fit parameter $\chi_{\nu}^{2}$ of the observed points is

$$
\chi_{\nu}^{2}=\frac{1}{n} \sum_{i=1}^{n} \frac{\left(X_{i}-\mu_{i}\right)^{2}}{u_{\mathrm{obs}}^{2}+u_{\text {intrinsic }}^{2}}
$$

where $n$ is the number of measured stars, $X_{i}$ is the measured abundance ratio in star $i, \mu_{i}$ is the average of all $X_{i}$, and again $u_{\text {obs }}$ is the observational uncertainty and $u_{\text {intrinsic }}$ represents the real, physical scatter in the abundance ratios. (A priori we do not expect a normal distribution, so we do not use $\sigma$ to 
represent the scatter in the denominator.) If there is a real intrinsic scatter in the stars, correctly estimated errors should not be able to account for all the observed dispersion. For each element and for each star, there is a quoted observational error $u_{\mathrm{obs}}$, so all variables except $u_{\text {intrinsic }}$ are fixed. We vary the intrinsic scatter term until $\chi_{\nu}^{2}=1$ (De Silva et al., 2006; Stuart and Ord, 2009). If $\chi_{\nu}^{2}>1$ without a nonzero $u_{\text {intrinsic }}$ term, this would mean there is more dispersion in the data than can be accounted for by observational errors alone. For $\chi_{\nu}^{2} \leq 1$, the spread for that element might be entirely explained by the observational error, although an intrinsic variation may yet exist at a small value hidden in generously estimated observational uncertainties. The inclusion of the intrinsic uncertainty term in this formulation also allows us to avoid unwanted emphasis on a few well-measured points.

Table 1 summarizes the calculated values of $u_{\text {intrinsic }}$ for six different abundance studies (Bond et al., 2006, 2008; Ramírez et al., 2007; Takeda, 2007; Mishenina et al., 2008; Neves et al., 2009; González Hernández et al., 2010). Of these studies, only Bond et al. (2006, 2008), González Hernández et al. (2010), Ramírez et al. (2007), and Neves et al. (2009) gave errors for individual stars. The other data sets gave the average error per element for the entire study. This lessens the robustness of this statistical method, as $u_{\text {intrinsic }}$ directly links observational error to spread on a star-to-star basis; use of an average error still allows for the calculation and comparison of $u_{\text {intrinsic }}$ but only provides a lower limit. Studies with larger observational errors, such as that of Delgado Mena et al. (2010), tend to not exhibit a $u_{\text {intrinsic }}$ value.

The relative pattern of $u_{\text {intrinsic }}$ between elements is consistent across all studies that yield a useful $u_{\text {intrinsic }}$. For example, $\mathrm{C}, \mathrm{Na}, \mathrm{Si}, \mathrm{Ca}$, and $\mathrm{Cr}$ have lower $u_{\text {intrinsic }}$ than other elements below the Fe peak, in all cases. Few studies look at elements above the Fe peak produced by the $r$ - and $s$ processes, but those that do find that heavier elements tend to have a large spread. Likewise, all groups tend to find a larger spread for $\mathrm{O}, \mathrm{Mg}$, and $\mathrm{Al}$. This seems to indicate that these elements have the largest actual scatter, but it must be noted that the larger $u_{\text {intrinsic }}$ for oxygen may be attributable to the difficulty in measuring $\mathrm{O}$ absorption lines and to a discrepancy in measurements due to the $6300 \AA$ forbidden line (Nissen, 2013). This line is strongly affected by a blend with a line of Ni I with strength $\sim 55 \%$ of the $\mathrm{O}$ I line in the Sun. At higher metallicities or lower abundances of $\mathrm{O}$ (relative to Fe-peak elements), the contribution can be even larger. The [O I] line at $6363 \AA$ also has a small contribution from a CN molecular line in cool stars (Caffau et al., 2013) but is generally considered more reliable than the $6300 \AA$ line. The O I triplet at $7774 \AA$ is not affected by blends but is sensitive to non-local thermodynamic equilibrium effects (Takeda, 2003; Ramírez et al., 2007; Fabbian et al., 2009). From our intrinsic variation analysis, we expect reliable results only from the $6363 \AA$ line and $7774 \AA$ triplet. To the extent that groups include the $6300 \AA$ line in their analysis, this may explain some of the apparently intrinsic scatter. The values quoted here refer to studies that do not use the $6300 \AA$ line.

Despite these caveats, the most striking fact about these abundances is that the light elements do show significant intrinsic variation. There are many stars that seem to have compositions significantly different from solar, by about a factor of 2 from the average in each direction.

\subsection{Planetary abundances (Nikku Madhusudhan)}

2.3.1. Abundances of gas giant exoplanets. Recently, it has become possible to estimate elemental abundance ratios in the atmospheres of "hot Jupiters," or highly irradiated

Table 1. Intrinsic Variations of Elemental Abundance Ratios for Different Abundance Studies

\begin{tabular}{|c|c|c|c|c|c|c|}
\hline \multirow[b]{2}{*}{ Element } & \multicolumn{6}{|c|}{ Intrinsic Variation } \\
\hline & Bond06\&08 & GH10 & Mish08 & Neves09 & Ramírez07 & Take07 \\
\hline $\mathrm{C}$ & 0.017 & 0.045 & & & & \\
\hline $\mathrm{O}$ & 0.092 & 0.052 & 0.153 & & $0.155(\mathrm{~L})$ and $0.197(\mathrm{~N})$ & \\
\hline $\mathrm{Na}$ & 0.036 & 0.034 & 0.013 & 0.052 & & 0 \\
\hline $\mathrm{Mg}$ & 0.068 & 0.029 & 0.116 & 0.054 & & 0.095 \\
\hline $\mathrm{Al}$ & 0.047 & 0.044 & 0.112 & 0.097 & & 0.085 \\
\hline $\mathrm{Si}$ & 0.014 & 0.014 & 0.062 & 0.040 & & 0.050 \\
\hline $\mathrm{S}$ & & 0.180 & & & & \\
\hline $\mathrm{Ca}$ & 0.016 & 0.007 & 0.065 & 0.028 & & 0 \\
\hline $\mathrm{Sc}$ & & 0.025 & & 0.090 & & 0.094 \\
\hline $\mathrm{Ti}$ & 0.026 & 0.002 & 0.061 & 0.083 & & 0.080 \\
\hline $\mathrm{V}$ & & 0 & 0.037 & 0.151 & & 0 \\
\hline $\mathrm{Cr}$ & 0.019 & 0 & 0.063 & 0 & & 0 \\
\hline $\mathrm{Mn}$ & & 0.057 & & 0.051 & & 0 \\
\hline Co & & 0.031 & 0.080 & 0.063 & & 0.028 \\
\hline $\mathrm{Ni}$ & 0 & 0.020 & 0 & 0.017 & & 0 \\
\hline $\mathrm{Cu}$ & & 0 & & & & 0 \\
\hline $\mathrm{Zn}$ & & 0.035 & 0.034 & & & 0 \\
\hline $\mathrm{Sr}$ & & 0.000 & & & & \\
\hline $\mathrm{Y}$ & 0.032 & 0.033 & & & & \\
\hline $\mathrm{Zr}$ & 0.113 & 0 & & & & \\
\hline $\mathrm{Ba}$ & 0.117 & 0.087 & & & & \\
\hline $\mathrm{Ce}$ & & 0.059 & & & & \\
\hline $\mathrm{Nd}$ & 0.033 & 0 & & & & \\
\hline $\mathrm{Eu}$ & 0.135 & 0.01 & & & & \\
\hline
\end{tabular}


gas giant exoplanets. Significantly, the $\mathrm{O} / \mathrm{H}$ and $\mathrm{C} / \mathrm{O}$ ratios are easier to measure for atmospheres of hot Jupiters than they are for Solar System giant planets. The vast majority of known extrasolar gas giants have $T \sim 600-3000 \mathrm{~K}$, thus hosting gaseous $\mathrm{H}_{2} \mathrm{O}$ in their upper atmospheres that is observable in their spectra. Other detectable gases include $\mathrm{CH}_{4}, \mathrm{CO}, \mathrm{CO}_{2}$, and $\mathrm{NH}_{3}$, depending on the temperature and incident irradiation (Burrows and Sharp, 1999; Lodders and Fegley, 2002; Madhusudhan, 2012). Measurements of these molecular abundances in the $\mathrm{H}_{2}$-rich atmospheres of hot Jupiters allow estimations of elemental abundance ratios involving $\mathrm{H}, \mathrm{C}, \mathrm{O}$, and $\mathrm{N}$. While a statistically significant constraint on the $\mathrm{C} / \mathrm{O}$ ratio of an exoplanetary atmosphere has been possible only recently (Madhusudhan et al., 2011a), nominal constraints on atmospheric $\mathrm{C}$, $\mathrm{O}$, and/or $\mathrm{C} / \mathrm{O}$ have been reported for several transiting exoplanets in the past decade (Fortney et al., 2005; Seager et al., 2005; Desert et al., 2009; Madhusudhan and Seager, 2009; Swain et al., 2009; Madhusudhan, 2012).

Beginning with the first inference of $\mathrm{C} / \mathrm{O}>1$ for WASP12b (Madhusudhan et al., 2011a), several other hot Jupiters are now suggested as possibly C-rich (Madhusudhan, 2012), though such inferences are still very preliminary given the low resolution of the data. Nevertheless, high $\mathrm{C} / \mathrm{O}$ ratios present a radical departure from our understanding of solar system chemistry. Most stars are oxygen-rich, with $\mathrm{C} / \mathrm{O}<1$ (Delgado Mena et al., 2010; Petigura and Marcy, 2011), like the Sun (C/ $\mathrm{O}=0.5$; Asplund et al., 2009). Assuming similar O-rich chemistry for gas giant planets, their nebular compositions would imply that $\mathrm{H}_{2} \mathrm{O}$ is the dominant molecule in their atmospheres and easily observable for hot Jupiters. At temperatures common to hot Jupiters $(T>1500 \mathrm{~K})$, and at $\mathrm{C} / \mathrm{O}$ ratios slightly higher than solar, the molecular chemistry in $\mathrm{H}_{2}$-rich atmospheres varies significantly from the familiar Solar System chemistry (Madhusudhan et al., 2011b; Kopparapu et al., 2012; Madhusudhan, 2012; Moses et al., 2013). For example, at $T=2000 \mathrm{~K}$, as the $\mathrm{C} / \mathrm{O}$ ratio is increased from the solar value of 0.5 to a $\mathrm{C}$-rich value of 1.1 , the $\mathrm{H}_{2} \mathrm{O}$ abundance drops by 2 orders of magnitude, and the $\mathrm{CH}_{4}$ abundance increases by over 3 orders of magnitude. Moreover, other hydrocarbons such as $\mathrm{C}_{2} \mathrm{H}_{2}$ and $\mathrm{HCN}$ also become markedly abundant for $\mathrm{C} / \mathrm{O}>1$, whereas $\mathrm{CO}_{2}$ becomes negligible. Such extreme changes in observable chemistry of hot Jupiters as a function of $\mathrm{C} / \mathrm{O}$ ratio make them ideal laboratories for constraining the elemental abundance ratios of $\mathrm{C} / \mathrm{H}, \mathrm{O} / \mathrm{H}$, and $\mathrm{C} / \mathrm{O}$.

Estimates of high $\mathrm{C} / \mathrm{O}$ ratios in giant exoplanets raise the question of how a C-rich gas giant could form around an $\mathrm{O}$ rich star, motivating new ideas on the formation of planetary systems. Lodders (2004) suggested the possibility that Jupiter, which the Galileo probe measured to have $\mathrm{C} / \mathrm{O}>1$ (at least locally: see Subsection 2.4), could have formed by accreting tar-dominated planetesimals instead of the water ice-dominated planetesimals expected in the Solar System based on the composition of minor bodies. Lodders (2004) suggested a high abundance of such planetesimals just beyond a "tar line" in the solar nebula (see also Subsection 3.2). In a similar vein, following the more recent inference of $\mathrm{C} / \mathrm{O}>1$ in WASP-12b, Öberg et al. (2011) suggested that $\mathrm{C} / \mathrm{O}$ ratios in giant planetary envelopes depend on the formation location of the planets in the disk relative to the condensation fronts, or ice lines, of major $\mathrm{C}$ - and O-bearing volatile species such as $\mathrm{H}_{2} \mathrm{O}, \mathrm{CO}$, and $\mathrm{CO}_{2}$, since the $\mathrm{C} / \mathrm{O}$ ratio of the gas approaches 1 outside the $\mathrm{CO}$ and $\mathrm{CO}_{2}$ ice lines. By predominantly accreting such $\mathrm{C}$-rich gas, more so than O-rich planetesimals, gas giants could, in principle, host $\mathrm{C}$-rich atmospheres even when orbiting O-rich stars. On the other hand, it may also be possible that inherent inhomogeneities in the $\mathrm{C} / \mathrm{O}$ ratios of the disk itself (e.g., by local concentrations of carbonaceous grains) may contribute to higher $\mathrm{C} / \mathrm{O}$ ratios of the planets relative to the host stars (Kuchner and Seager, 2005; Madhusudhan et al., 2011b; Mousis et al., 2012). Internal processes of the planets themselves may cause the composition of the observable parts of the atmosphere to inaccurately reflect the bulk composition (see also Subsection 2.4.1). These scenarios predict different limits on the metallicities and $\mathrm{C} / \mathrm{O}$ ratios of the giant planets, which high-precision observations of their atmospheres will be able to test in the near future but for which knowing the abundances of the host stars is a central requirement.

2.3.2. Abundances of rocky exoplanets. In contrast to gas giant planets, rocky planets form almost entirely from refractory condensates, possibly with some volatile condensates, but with very little gas accreted from the disk. Rocky planet compositions are governed by what minerals condense near the planet's location in a protoplanetary disk with distinct elemental composition. Bulk compositions for terrestrial-type planets are expected to span a wide range based on models (i.e., Carter-Bond et al., 2012) and on observations of our own solar system. The potential for forming systems with significantly nonsolar ratios is significant. Wide ranges of $\mathrm{C} / \mathrm{O}$ and $\mathrm{Mg} / \mathrm{Si}$ values have been observed in the photospheric abundances of exoplanet host stars. The frequency of very $\mathrm{C}$-rich stars $(\mathrm{C} / \mathrm{O}>0.8)$ is being debated, but some examples certainly exist, and large $\mathrm{Mg} / \mathrm{Si}$ variations are in little doubt (Delgado Mena et al., 2010, Petigura and Marcy, 2011, Fortney, 2012, Nissen, 2013).

The advent of high-precision measurements of masses and radii of transiting super-Earths (exoplanets with radii between 1 and 10 Earth radii) are making it feasible to place nominal constraints on the range of their possible interior compositions. Where available, the abundances of the host stars can significantly improve the constraints on the super-Earth compositions by constraining the mineralogy. Masses and radii are known for $\sim 15$ transiting super-Earths to date. Models of superEarths used to interpret masses and radii in the past have traditionally assumed Earth-like oxygen-rich interiors made of $\mathrm{Fe}$, silicates, and water (Valencia et al., 2007, 2010; Charbonneau et al., 2009; Rogers and Seager, 2010; Winn et al., 2011; Gillon et al., 2012). Recently the first inference of a C-rich super-Earth was reported for 55 Cancri e (Madhusudhan et al., 2012). The star was known to be carbon-rich (Delgado Mena et al., 2010), but the $\mathrm{C} / \mathrm{O}$ ratio of the system may be lower than originally thought (Teske et al., 2013). Using the planet's mass and radius, Madhusudhan et al. suggested a carbon-rich interior in the planet, with carbon (as graphite and diamond), iron, silicon carbide, and/or silicates as the primary constituents and water being scarce. The drastically different thermochemical properties of carbon-rich minerals open an entirely new regime of geological processes (see Subsection 3.4).

\subsection{Lessons from our solar system}

2.4.1. Inferring gas giant planet compositions (Steven Desch). Since the detection of $\mathrm{Na}$ in the atmosphere of HD 
209458b (Charbonneau et al., 2002), transmission spectroscopy has offered the promise of constraining the abundances of gas giant exoplanets. Important to note, however, is that such measurements probe only the upper atmospheres of gas giant planets and not necessarily their bulk abundances. The difficulties of translating observable abundances into bulk abundances are perfectly illustrated by the example of Jupiter in our own Solar System.

The Galileo entry probe's Neutral Mass Spectrometer measured the abundances of $\mathrm{H}, \mathrm{He}, \mathrm{C}, \mathrm{N}, \mathrm{O}, \mathrm{Ne}, \mathrm{S}, \mathrm{Ar}, \mathrm{Kr}$, and Xe in Jupiter's atmosphere, between the 0.88 and 21.7 bar levels (Niemann et al., 1998; Mahaffy et al., 2000; Wong et al., 2004). The abundance of $\mathrm{P}$ was measured remotely from the Galileo orbiter's Near Infrared Mapping Spectrometer (Irwin et al., 1998). In Fig. 3, we plot the elemental ratios $\mathrm{He} / \mathrm{H}, \mathrm{C} / \mathrm{H}$, and so on, normalized to the values in the solar nebula (Asplund et al., 2009). The species $\mathrm{C}, \mathrm{N}, \mathrm{S}, \mathrm{P}$ and the noble gases $\mathrm{Ar}, \mathrm{Kr}$, and $\mathrm{Xe}$ are all enriched relative to solar by a similar factor of 3 . He, in contrast, is depleted by $15 \%$ below the protosolar value, and $\mathrm{Ne}$ is depleted by a factor of $6 . \mathrm{O}$ is depleted by a factor of 2 .

The enrichment of most species is potentially explained by external photoevaporation of the Sun's protoplanetary disk by far-ultraviolet (FUV) radiation from a nearby massive star

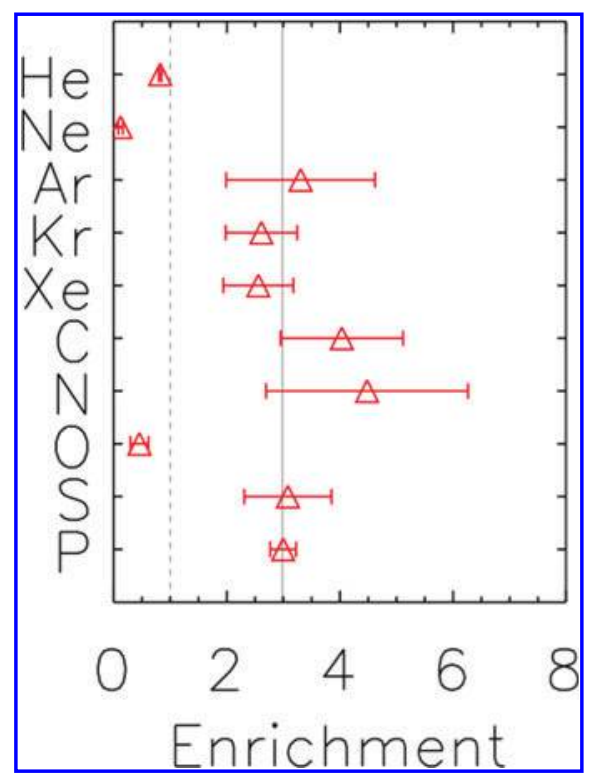

FIG. 3. Abundances of elements in the upper atmosphere of Jupiter, as measured by the Galileo entry probe and orbiter. Abundances are reported relative to $\mathrm{H}$ abundance and relative to solar composition (Asplund et al., 2009). C, N, S, $\mathrm{P}$, and even $\mathrm{Ar}, \mathrm{Kr}$, and $\mathrm{Xe}$ are apparently uniformly enriched by a factor of 3 above solar. He is depleted, presumably because of $\mathrm{H} / \mathrm{He}$ demixing at megabar pressures. $\mathrm{Ne}$ is depleted by a factor of 6, presumably because of sequestration into precipitated He droplets. Galileo measured a factor of 2 depletion in $\mathrm{O}$, but it was argued to be a local anomaly. Alternatively, this may be evidence that $\mathrm{O}$ was enriched by a factor of 3 like other species, then depleted by a factor of 6 like $\mathrm{Ne}$, due also to sequestration into precipitated He droplets. Surface measurements of O may not reflect bulk abundances. Color images available online at www.liebertonline.com/ast
(Guillot and Hueso, 2006). Within a thin $\left(<0.1 \mathrm{~g} \mathrm{~cm}^{-2}\right)$ layer, gas is heated to temperatures $\approx 500-1000 \mathrm{~K}$, sufficient to drive the gas to escape the gravitational pull of the star. If the FUV radiation is propagating into gas that normally is at temperatures $<25 \mathrm{~K}$, it is possible that only $\mathrm{H}_{2}, \mathrm{He}$, and $\mathrm{Ne}$ remain in the gas phase, as even $\mathrm{N}_{2}$ and Ar can be trapped in amorphous water ice at these cold temperatures (Bar-Nun et al., 1985; Owen et al., 1992). Although Guillot and Hueso (2006) only considered photoevaporation off the top and bottom surfaces of the disk, where temperatures probably are not $<25 \mathrm{~K}$, Adams et al. (2004) showed that when considering a full disk, most mass loss will take place at an outer edge of the disk, $r_{\mathrm{d}}$. For typical FUV fluxes $\left(G_{0}=3000\right.$ in Habings, $1.6 \times 10^{-3} \mathrm{erg} \mathrm{cm}^{-2} \mathrm{~s}^{-1}$, equivalent to an $\mathrm{O} 6 \mathrm{star}$ at $2 \mathrm{pc}$ ) and typical protoplanetary disk parameters, $r_{\mathrm{d}}=50 \mathrm{AU}$ (Adams et al., 2004). (This is also the location of the edge of the Solar System's Kuiper belt: Morbidelli et al., 2003.) At this distance in a protoplanetary disk, temperatures are cold enough that all species except $\mathrm{H}, \mathrm{He}$, and $\mathrm{Ne}$ are likely to be trapped in icy bodies and not removed with the photoevaporated gas. Jupiter easily could have accreted from gas that was not so much enriched in the heavier species but depleted in $\mathrm{H}$.

Loss of $\mathrm{H}_{2}, \mathrm{He}$, and $\mathrm{Ne}$ from a photoevaporated disk explains why other species are enriched but does not explain the depletions in $\mathrm{He}$ and $\mathrm{Ne}$. The leading explanation for these depletions is demixing at high pressures. At depths of several thousand kilometers and pressures of several megabar, $\mathrm{H}_{2}$ is predicted to transition from a molecular gas to metallic $\mathrm{H}$, separating out from $\mathrm{He}$ in the process. He droplets must form that sink because they are denser than the surrounding fluid. Precipitation of He to Jupiter's core over many billions of years is thought to be the cause of Jupiter's He depletion (Stevenson and Salpeter, 1977a, 1977b). It is hypothesized that Ne would partition into the He droplets but Ar would not, a result qualitatively confirmed by Wilson and Militzer (2010) using density functional theory-molecular dynamics calculations.

This leaves only the case of $\mathrm{O}$ as anomalous. Soon after the Galileo results showed that other species were enriched but $\mathrm{O}$ was depleted, it was suggested that the probe sampled a water-poor region of Jupiter. This explanation is very plausible given that the probe entered one of Jupiter's " $5 \mu \mathrm{m}$ hotspots," downwellings of gas analogous to high-pressure cells, from which water has been removed (Atreya et al., 1999). The Juno mission, arriving at Jupiter in 2016, will globally probe the $\mathrm{O} / \mathrm{H}$ ratio down to the 200 bar level to test this hypothesis (Matousek, 2007).

An alternative is that Jupiter accreted gas with an $\mathrm{O} / \mathrm{H}$ ratio 3 times solar, and then depleted by a factor of 6 similar to $\mathrm{Ne}$, ending up with a factor of 2 depletion. Dissolution of $\mathrm{Ne}$ but not Ar into He droplets is controlled to a large degree by atomic radius (Wilson and Militzer, 2010). N, C, Ar, Kr, and $\mathrm{Xe}$ have atomic radii larger than that of $\mathrm{H}$, while $\mathrm{He}$ and $\mathrm{Ne}$ have atomic radii smaller than $\mathrm{H}$ (Clementi et al., 1967). The atomic radius of $\mathrm{O}$ is not as small as $\mathrm{Ne}$, but it is smaller than $\mathrm{H}$ and may be expected to dissolve in the He droplets as well. If Juno measures a global depletion in $\mathrm{O} / \mathrm{H}$, it will be difficult to interpret the result in any way except that $\mathrm{O}$, like $\mathrm{He}$ and $\mathrm{Ne}$, is sequestered in Jupiter's core.

$\mathrm{H}_{2} \mathrm{O}$ and $\mathrm{CH}_{4}$ are some of the species most easily measured by transmission spectroscopy, and the $\mathrm{C} / \mathrm{O}$ ratio that can be derived from these measurements is crucial to 
chemical models of planet formation. But there are no guarantees that measurements of a gas giant planet's upper atmosphere will reflect its bulk abundance or the star's abundance. If $\mathrm{O}$ can be sequestered in a gas giant planet, transmission spectroscopy of its upper atmosphere will overestimate the $\mathrm{C} / \mathrm{O}$ ratio.

2.4.2. Inferring terrestrial planet compositions (Miriam Riner). Beginning just in 2009, it has become possible to measure the mass and radius of exoplanets not much more massive than Earth, such as CoRoT-7b (Queloz et al., 2009) and GJ 1214b (Charbonneau et al., 2009 and subsequent papers). With the mass and radius known, it is possible to estimate the density and thereby infer the bulk composition. In practice, what are published are mass-radius relationships (e.g., Fortney et al., 2007; Seager et al., 2007; Barnes et al., 2013). In these plots, individual curves of radius versus mass are computed for an assumed composition (or at most two compositions, one in a core and one in a mantle). The mass-radius error ellipses of exoplanets are plotted over these lines to infer their compositions. Here "composition" refers to the relative abundance of iron metal, rock, and water ice. Through use of such plots, it has been inferred that Kepler-11b is likely to be more water ice than silicate rock, and CoRoT-7b is more likely to be more Fe metal than silicate rock (Kaltenegger et al., 2013).

These models of mass-radius relationships build on those developed for studying planets in our own solar system, inheriting their insights and their built-in assumptions. We must understand how simplifying assumptions and uncertain input parameters translate into uncertainties in the massradius relationships and inferred compositions.

Mass-radius relationships must be computed because materials under planetary interior pressures change density. This is done by using laboratory-determined properties of the materials assumed for a metallic core, rocky mantle, and/or icy layer, together with an adopted equation of state (EOS), to compute the effects of self-compression on the interiors of model planets. Different approaches vary in detail based on the adopted EOS and other assumptions. As an example, Riner et al. (2008) modeled the self-compression of the Solar System terrestrial planets, especially Mercury, using the Birch-Murnaghan finite strain EOS and assuming an adiabatic temperature profile. Planets are divided into multiple layers within which material properties are assumed to be uniform. For each material or layer, the model requires the density of the material at standard temperature and pressure $(\rho)$, the bulk modulus $(K)$, the pressure derivative of the bulk modulus $\left(K^{\prime}\right)$, the thermal expansion coefficient $(\alpha)$, and the second Grüneisen parameter $(\delta$, which describes the relationship between bulk modulus and density). By using these material properties, together with an assumed temperature difference across thermal boundary layers where the composition changes, the effect of self-compression on the volume of a planet of a given composition can be computed, leading to mass-radius relationships.

Because a composition is assumed, then a mass-radius relationship calculated, there are several limits to using such relationships to infer internal composition; a single massradius pair does not uniquely constrain the composition of a planet. The mass and radius of Mercury have been accurately known for six decades (Urey, 1951), yet its metal-to- silicate ratio (or the percent of mass in the core) was poorly known until new spacecraft measurements of its moment of inertia. Until recently, published estimates of the core mass fraction ranged anywhere from $50 \%$ to $100 \%$ of the total planetary mass (Harder and Schubert, 2001; Chen et al., 2007; Riner et al., 2008).

Much of this uncertainty has stemmed from poor knowledge of the composition (abundance and identity of a light-alloying element) and phase (liquid or solid) of the core. For example, liquid $\mathrm{Fe}$ is $20 \%$ less dense and $40 \%$ more compressible than solid iron (face-centered cubic phase). While these differences tend to diminish at higher pressures, uncertainties in material properties also are greater at higher pressures. To a lesser degree, uncertainties in mantle composition also matter. The major mantle compositional variation relevant to mass-radius relationships in our Solar System is the relative abundance of Fe and $\mathrm{Mg}$ in the mantle (the Mg\#). While the Mg\# makes a substantial $(\sim 40 \%)$ difference in density, the compressibility and other material properties vary less than those due to compositional variations in core materials. That said, the uncertainty in the mass-radius relationship due to variations in $\mathrm{Mg \#}$ has not been documented over a wide range of planetary masses or $\mathrm{Mg \#}$ variations.

Additional uncertainty stems from the difficulty of measuring EOS data. The material properties of candidate compositions at planetary interior pressures and temperatures must be determined through static diamond-anvil cell experiments (for pressures up to $\sim 100 \mathrm{GPa}$, equivalent to Earth's core mantle boundary) or shock-wave experiments (for pressures up to $\sim 300 \mathrm{GPa}$, Earth's inner core but temperature is not controlled). Mass-radius relationships can only be constructed assuming those materials that have been measured in these ways.

The level of uncertainty about the composition and structure of our own planet, and the degree to which other sources of information are used, is often underappreciated by scientists working outside the field in astrophysics or planetary science. From seismic measurements, we know that the density of the molten outer core of Earth requires the presence of one or more light-alloying elements. Usually this element is assumed to be $\mathrm{S}$, in part because data are lacking for other candidate alloys ( $\mathrm{H}$ or $\mathrm{Si}$ in $\mathrm{FeNi}$ ). Although composing only a few percent of the core's mass, this alloying element can make a substantial difference in the material properties of the core and therefore the assumed bulk composition of the planet. In practice, we use cosmochemical abundances measured in meteorites and the solar atmosphere to make reasonable assumptions about the identity of the light alloying element.

The assumptions we typically make for our Solar System may not be valid in other stellar systems. Sulfur can be redistributed during planet formation, and rocky exoplanets may have different $\mathrm{S}$ abundances in their cores, affecting our interpretation of their bulk rock/metal ratio. The observed variations in stellar $\mathrm{Mg} / \mathrm{Si}$ ratios, if acquired by these stars' planets, may introduce high-pressure mineral phases in their mantles that do not exist in Earth's (as discussed in Subsection 3.4).

To make substantial progress in deriving accurate massradius relationships for exoplanets, it will be necessary to experimentally measure a greater range of material 
properties spanning the potential compositional variability. At the same time, the uncertainties in input parameters and the limitations of mass-radius relationship modeling should be understood, especially by astronomers, to avoid overly interpreting the observational data.

\section{Effects of Composition on Stars and Planets}

Even though virtually all stars are overwhelmingly ( $>98 \%$ by mass) $\mathrm{H}$ and He, a star's evolution will be significantly affected by the abundances of those trace $(<2 \%)$ elements heavier than He. Modeling the effect of composition on stars is essential but is a relatively simple problem compared to planets. The structure and internal processes of a planet are, of course, completely changed depending on its composition: whether it is a gas giant, Neptune-sized ice giant, or a rocky planet, or simply subtle variations of these classes.

As can be expected, the habitability of a planet is even more sensitive to the composition of the planet itself. The ability to keep liquid water on an Earth-like planet's surface (the usual criterion of habitability) depends on the atmosphere of the planet, which in turn depends on its composition. There are also composition-dependent processes internal to an Earth-like planet that can affect its surface, such as radiogenic heating, internal mineralogy and rheology, thermal evolution, and convective mixing.

\subsection{Abundance variations and stellar and habitable zone evolution (Patrick Young)}

Models of the evolution of stars of different metallicity are generally created assuming the heavy elements are found in the same proportions relative to $\mathrm{Fe}$ as in the Sun. Except for uniform enhancement of the $\alpha$ elements $(\mathrm{O}, \mathrm{Ne}, \mathrm{Mg}, \mathrm{Si}$, $\mathrm{S}, \mathrm{Ar}, \mathrm{Ca}, \mathrm{Ti})$ in stars of very low metallicities, variations in abundance ratios at a given $[\mathrm{Fe} / \mathrm{H}]$ are neglected, despite the fact that stellar evolution is sensitive to the star's composition.

The sensitivity to composition arises from two effects: the EOS and the radiation opacity (Iglesias and Rogers, 1996; Rogers et al., 1996). The changes in the EOS are relatively minor, but rearranging the proportions of different species at a constant $[\mathrm{Fe} / \mathrm{H}]$ can result in opacity changes of tens of percent (Iglesias and Rogers, 1996). Opacity changes the rate of leakage of radiation, and increased radiation pressure in the stellar envelope drives expansion, resulting in larger radii and lower effective temperatures. A slower rate of energy loss also requires a slower rate of nuclear burning to maintain hydrostatic equilibrium. So we expect stars with enhanced abundances to be cooler, of lower luminosity, and longer-lived relative to stars with the same $[\mathrm{Fe} / \mathrm{H}]$.

The large observed intrinsic spread in $[\mathrm{O} / \mathrm{Fe}]$ (see Subsection 2.2) and the high abundance of $\mathrm{O}$ make this element particularly interesting. In stellar interiors, it is second only to Fe in contributing to the opacity. Stellar evolution simulations [herein using the TYCHO code (Young and Arnett, 2005)] with variable abundance ratios show that the impact is dramatic (Young et al., 2012).

In these models, a solar composition from the work of Lodders (2010) was adjusted to the mean abundance ratios of the sample in Subsection 2.2, with all other elements being maintained at solar values for the standard composition. The intrinsic physical spreads $u_{\text {intrinsic }}$ in the element-
to-Fe ratios are the basis for the variant models. In each model, one element was varied to the $3 u_{\text {intrinsic }}$ extremes of the distribution, resulting in one standard composition model and two extreme models for each of the elements $\mathrm{C}$, $\mathrm{O}, \mathrm{Na}, \mathrm{Mg}, \mathrm{Al}, \mathrm{Si}, \mathrm{Ca}$, Ti. Custom opacity tables for each composition were obtained from the OPAL project (Iglesias and Rogers, 1996). All models were run until core hydrogen exhaustion. The models provide high time-resolution histories of luminosity and effective temperature.

The prescription of Kopparapu et al. (2013), a conceptual descendant of Kasting et al. (1993), was used to convert modeled stellar effective temperature $T_{\text {eff }}$ and luminosity versus time into $\mathrm{HZ}$ boundaries. This treatment allows the $\mathrm{HZ}$ edges to be easily scaled from the stellar quantities for different assumptions, and the relative differences between the stellar models are robust.

In all cases, enhanced abundance ratios cause larger changes than depletions. $\mathrm{C}, \mathrm{Na}$, and $\mathrm{Mg}$ have small but noticeable effects. $\mathrm{C}$ has a high abundance but relatively few electron transitions and low ionization potential. $\mathrm{Mg}$ and $\mathrm{Na}$ have lower abundances but higher opacity per gram than $\mathrm{C}$, resulting in a similar degree of shift in the tracks. Si has less impact due to its smaller range of variation, and $\mathrm{Al}, \mathrm{Ca}$, and Ti have very small effects due to their small abundances; these can be neglected for the purpose of habitability. The largest changes, unsurprisingly, arise from variation in $\mathrm{O}$. Both the luminosity and $T_{\text {eff }}$ of enriched compositions are systematically lower at a given age, but the most profound effect is on the pace of the evolution. The main-sequence turnoff for the low-[O/Fe] model occurs at an age of $9 \mathrm{Gyr}$. The standard composition (which is slightly more O-rich than solar) has a turnoff age of $10.5 \mathrm{Gyr}$, and the O-rich model turns off at $12 \mathrm{Gyr}$ (and at lower luminosity and $T_{\text {eff }}$ ).

This time evolution of the $\mathrm{HZ}$ is highly significant. If the ultimate goal is the discovery of life on an Earth-like world, one must consider not just the current habitability of the planet but also how long the planet has remained in its HZ. Life did not modify Earth's atmospheric composition at levels that might be detectable by near-future astronomical observations until about a billion years after its formation. Complex life on Earth developed during the late Ediacaran period, 635-542 Myr ago, some 4Gyr after its formation. Orbits with semimajor axes near the edges of HZs will not remain there for the entire lifetime of the star. Figure 4 shows the inner and outer edges of HZs for high, low, and standard $[\mathrm{O} / \mathrm{Fe}]$ as a function of time, calculated by using conservative and optimistic cases chosen from the parameterization of Kopparapu et al. (2013). The dashed line marks a 1 AU orbit. The conservative case (top) uses the prescriptions for the Runaway and Maximum Greenhouse models. The optimistic case (bottom) uses the Recent Venus and Early Mars values.

For a star otherwise identical to the Sun in mass and $\mathrm{Fe}$ abundance at the lower end of the range of [O/Fe] observed in planet host candidates, an Earth-like planet at $1 \mathrm{AU}$ from the star would not (given the assumptions about planetary atmospheres herein) remain habitable long enough for complex life to have developed at the pace that occurred on Earth. The effect is obviously dramatic for the extremes of $\mathrm{O}$ variation, but the effect could be important to less extreme systems with planets on the edges of their nominal HZs. For a set of stars deliberately chosen to be maximally Sun-like by Ramírez et al. (2009), the smaller range of $[\mathrm{O} / \mathrm{Fe}]$ still causes the $1 \mathrm{AU}$ habitability 


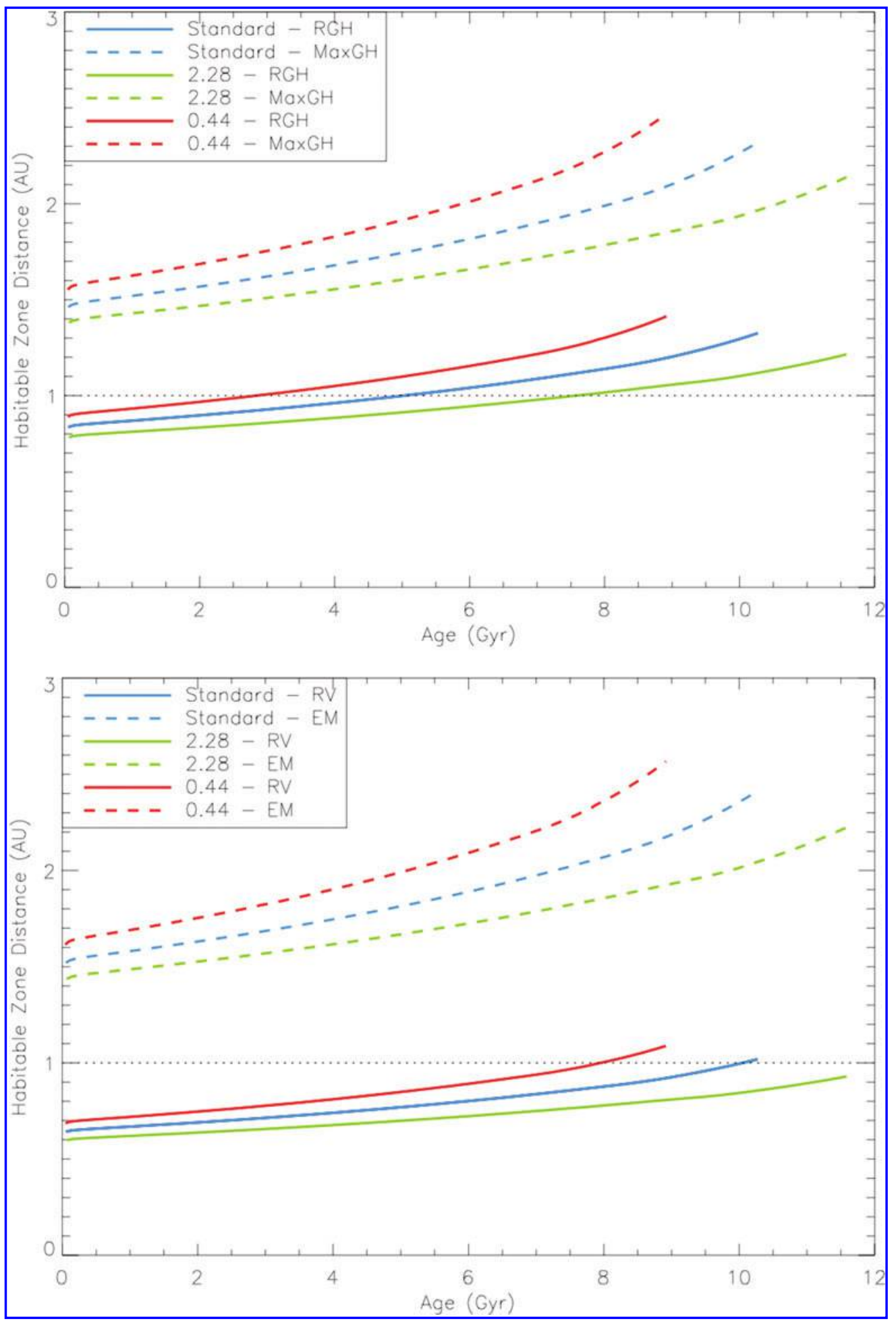

FIG. 4. Inner and outer edges of the $\mathrm{HZ}$ versus time for variations in $\mathrm{O} / \mathrm{Fe}$ for conservative (top) and optimistic (bottom) HZ predictions. Habitable zones are shown for models with depleted (upper; red), standard (middle; blue), and enhanced (lower; green) O abundance ratios. Inner edges are solid; outer edges are dashed. A 1 AU orbit is indicated by the dashed horizontal line. The conservative case uses the prescriptions for the Runaway (RGH) and Maximum (MaxGH) Greenhouse cases of Kopparapu et al. (2013). The optimistic case uses the recent Venus (RV) and early Mars (EM) values. Depletions systematically move the HZs outward due primarily to increased luminosity and secondarily to increased $T_{\text {eff. }}$ The difference in location increases markedly with age. The most dramatic effect results from the substantial difference in main sequence lifetimes. Color images available online at www.liebertonline.com/ast 
lifetime to differ by nearly a billion years. The magnitudes of the effect for the observed ranges of $[\mathrm{C} / \mathrm{Fe}],[\mathrm{Na} / \mathrm{Fe}]$, and $[\mathrm{Mg} /$ $\mathrm{Fe}]$ are hundreds of millions to a billion years.

Only a very limited range of orbits remain continuously habitable. For the conservative atmospheric prescription, there are no orbits continually habitable for all the models. Even the most optimistic case considered has orbits that are continuously habitable for all compositions extending only from 1.1 to 1.4 AU. Ranges are similarly narrow, though shifted in radius, for other $[\mathrm{Fe} / \mathrm{H}]$. The range of orbits that provide at least $4 \mathrm{Gyr}$ of continuous habitability are much more extensive. High- $[\mathrm{O} / \mathrm{Fe}]$ models have the widest range of 4 Gyr habitable orbits by a small margin due to their longer overall main sequence lifetime and shallower rate of change of the zone boundaries. A point that is not commonly considered is that the majority of orbits that are at some point habitable only become so during the latter part of the star's lifetime. While these abundance variations encompass the majority of nearby planet host candidates, there are even more extreme outlier systems. The stars HD $53705([\mathrm{O} / \mathrm{Fe}]=$ $0.6)$ and HD 136352 ([C/Fe] also 0.6) have a substantially larger range of orbits that remain habitable for a multi-billion year window due to their large enhancements.

The stellar characteristics that determine the size and persistence of HZs change at an important level with different abundance ratios. Therefore proper characterization of a system requires more data than just $[\mathrm{Fe} / \mathrm{H}]$.

\subsection{Habitable zones from atmospheric models and the frequency of potential habitable planets in extrasolar planetary systems (Ravi Kopparapu)}

The HZ is classically defined as the circumstellar region in which a terrestrial-mass planet $\left(0.1 \leq M_{\mathrm{p}} \leq 10 M_{\text {Earth }}\right)$ with favorable atmospheric conditions can sustain liquid water on its surface (Huang, 1959; Hart, 1978; Kasting et al., 1993; Selsis et al., 2007; Kopparapu et al., 2013). Liquid water is often mentioned as a prerequisite for life, and this restriction is appropriate for remote detection of life on planets around other stars. Life as we know it is carbon-based and depends on liquid water.

The average temperature on Earth's surface is approximately $289 \mathrm{~K}$, set by the flux of radiation received from the Sun and also by our atmosphere. On an airless body with the same albedo as Earth (0.3), 1 AU from our sun, the blackbody surface temperature would be only $255 \mathrm{~K}\left(-18^{\circ} \mathrm{C}\right)$, incompatible with liquid water, at least on the surface of the planet. More importantly in that case, of course, liquid water is not stable at any temperature at sufficiently low atmospheric pressure. The atmospheric greenhouse effect, which is attributable mostly to $\mathrm{H}_{2} \mathrm{O}$ and $\mathrm{CO}_{2}$, completely changes the habitability of a planet and the $\mathrm{HZ}$ boundaries.

Following Kopparapu et al. (2013), and assuming an Earth-like planet and atmospheric composition, two kinds of HZ limits are considered:

(1) Conservative HZ: The inner edge of the $\mathrm{HZ}$ in this limit is fixed by the moist-greenhouse effect, which is reached once the surface temperature exceeds $\sim 340 \mathrm{~K}$ (for a 1 bar Earth-like atmosphere) and the $\mathrm{H}_{2} \mathrm{O}$ saturation mixing ratio at the surface exceeds 0.2 . This results in a large increase in the altitude of the tropopause and a corresponding increase of water vapor in the stratosphere. For
Earth, the moist-greenhouse limit is $0.99 \mathrm{AU}$ (or in terms of stellar flux normalized to the flux at Earth's orbit, $S_{\text {eff }}=$ 1.014) (Kopparapu et al., 2013). If the surface temperature exceeds $647 \mathrm{~K}$ (the critical temperature for pure $\mathrm{H}_{2} \mathrm{O}$ ), the entire ocean should evaporate. This describes the runaway greenhouse limit, which is reached at $0.97 \mathrm{AU}\left(S_{\text {eff }}=1.038\right)$ for Earth. The outer edge of the HZ is determined by the maximum greenhouse limit, which is the distance at which increasing $\mathrm{CO}_{2}$ partial pressures in the atmosphere would no longer result in additional greenhouse warming; increasing $\mathrm{CO}_{2}$ partial pressures would tend to lead to surface cooling (Kasting et al., 1993; Kopparapu et al., 2013). For Earth, the maximum greenhouse limit is at $1.68 \mathrm{AU}\left(S_{\mathrm{eff}}=0.35\right)$.

(2) Optimistic HZ: For the inner edge of the HZ in our solar system, an empirical estimate can be calculated based on the observation that Venus could have had significant water up to as late $1 \mathrm{Gyr}$ ago. The Sun at that time was $\sim 8 \%$ less bright than it is today. Therefore, we can derive a recent Venus limit for the inner edge, which is at $0.75 \mathrm{AU}\left(S_{\mathrm{eff}}=\right.$ 1.77). Similarly, an empirical estimate for the outer edge of the HZ can also be derived based on the observation that Mars appears to have had liquid water flowing on its surface at or before $3.8 \mathrm{Gyr}$ ago, when the solar flux was some $25 \%$ lower (Kopparapu et al., 2013). This early Mars limit corresponds to a distance of $1.76 \mathrm{AU}\left(S_{\text {eff }}=0.32\right)$.

Parametric forms of both the conservative and optimistic estimates of the $\mathrm{HZ}$ for stars of different spectral types were given by Kopparapu et al. (2013) and are displayed in Fig. 5 . Using these estimates, and six quarters of Kepler data, Kopparapu et al. (2013) showed that the occurrence rate of terrestrial-sized $\left(0.5-2 R_{\text {Earth }}\right)$ planets around $\mathrm{M}$ dwarfs can vary from $48 \%$ (conservative estimate) to as high as $61 \%$ (optimistic estimate). So the potential for finding Earth-like planets around $\mathrm{M}$ dwarfs may be higher than previously reported (Dressing and Charbonneau, 2013).

It should be noted that the HZ estimates discussed above used 1-D radiative-convective climate models that do not include cloud feedback. Results from 3-D global climate models that do include cloud behavior (Abe et al., 2011; Wordsworth et al., 2011; Yang et al., 2013) show that the $\mathrm{HZ}$ may be wider; hence the terrestrial planet occurrence rate quoted above may be larger than the previous estimates. This bodes well for future discovery missions like the Transiting Exoplanet Survey Satellite and for future characterization missions such as the James Webb Space Telescope as they attempt to find the first habitable planet outside our Solar System, at least for planets with atmospheric compositions like Earth's.

The distinct possibility exists that an exoplanet may have Earth's mass and orbit 1 AU from a Sun-like star yet not be habitable, because its atmospheric composition is completely different. Earth's atmosphere contains $4 \times 10^{-4}$ bar of the major greenhouse gas $\mathrm{CO}_{2}$ but would contain tens of bars of $\mathrm{CO}_{2}$ without the carbonate-silicate cycle. The same assumptions that optimistically place the outer edge of the $\mathrm{HZ}$ at $1.76 \mathrm{AU}$ could also involve an inner edge beyond 1.0 AU. Enhanced atmospheric $\mathrm{CO}_{2}$ abundance could be due to increased volatile abundance on the planet or to more subtle transport processes. Conversely, in a system with high $\mathrm{C} / \mathrm{O}, \mathrm{C}$ will preferentially be incorporated into other species. A highly reducing environment may be dominated by $\mathrm{CH}_{4}$, a more effective greenhouse gas than $\mathrm{CO}_{2}$. 


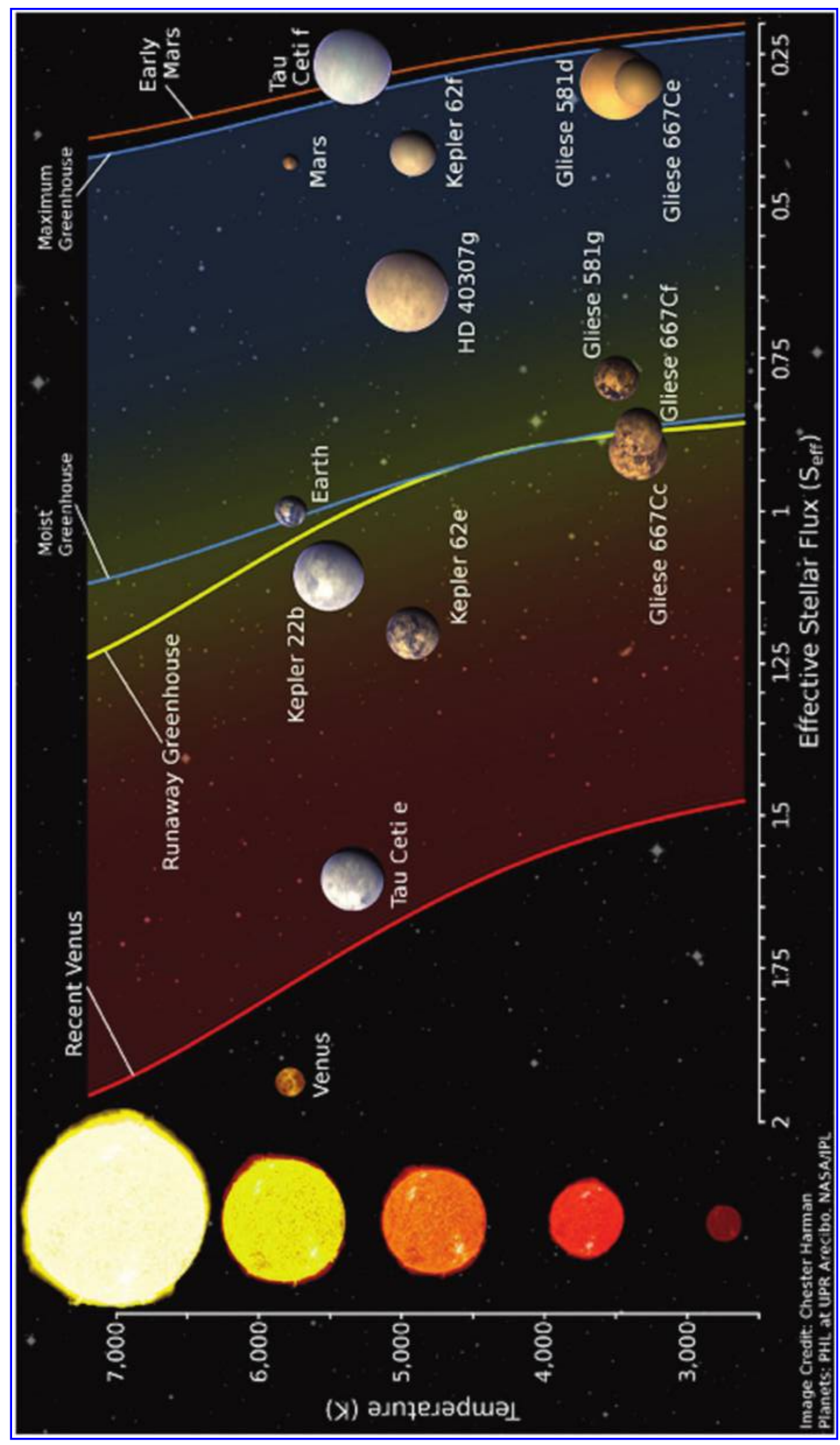

을 일

$\therefore$ 응.

들 응

¿

스늘 을

嗐

ซึ สิ ซृ

记造

웅

ปृ

的

츨

年的

苟茟

를 至

을

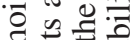

(.) 0.

Ð $\approx$

Nㅗ콕

莫司焉

을. 졸

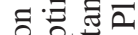

क्ष 의

.

F

거 운

它 0 क ज्ञ

$\checkmark \cong$

눙웡

氜苛

¿.

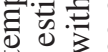

0 ¿

츨

巴ै

ข

층 0

ब

西结.

ธี

울웜

(1) के

․․․

๙

๑ $\Xi \stackrel{2}{\square}$

를늉

츙 증

๙

드릉 엉은

뜰.전

든

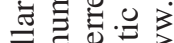

到

릉

후요

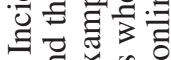
텄 a ம่)

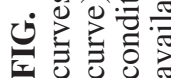


We must consider more than a potentially very different primordial volatile inventory. The surface exchange and mantle recycling processes that modify atmospheres may operate very differently than on Earth if the solid rock geochemistry is exotic. It is difficult to overstate the importance of chemical composition on the contribution of atmospheres to habitability, an area that is largely unexplored at this time.

\subsection{Internal heating of terrestrial exoplanets (Rory Barnes)}

On Earth, plate tectonics and the hydrologic cycle combine to create the carbonate-silicate cycle that prevents the secular buildup of $\mathrm{CO}_{2}$ in the atmosphere, staving off a sterilizing moist greenhouse (Walker et al., 1981). Without an internal energy source to sustain plate tectonics, Earth may have become a barren wasteland by today.

Three primary sources of energy are postulated: latent heat from formation, gravitational energy released by differentiation, and radiogenic heating from the decay of radioactive isotopes. Beyond Earth, tidal heating provides significant energy on several icy satellites (see, e.g., Peale et al., 1979; Hurford et al., 2007) and may be important for planets in the HZ of low-mass stars (Jackson et al., 2008; Barnes et al., 2013).

The primary sources of radiogenic energy in Earth are ${ }^{40} \mathrm{~K}$, U, and Th. Their decay chains are

$$
\begin{aligned}
{ }^{238} \mathrm{U} & \rightarrow{ }^{206} \mathrm{~Pb}+8 \alpha+6 \mathrm{e}^{-}+6 \bar{\nu}_{\mathrm{e}}+51.698 \mathrm{MeV} \\
{ }^{235} \mathrm{U} & \rightarrow{ }^{207} \mathrm{~Pb}+7 \alpha+4 \mathrm{e}^{-}+4 \bar{\nu}_{\mathrm{e}}+46.402 \mathrm{MeV} \\
{ }^{232} \mathrm{Th} & \rightarrow{ }^{208} \mathrm{~Pb}+6 \alpha+4 \mathrm{e}^{-}+4 \bar{\nu}_{\mathrm{e}}+42.652 \mathrm{MeV} \\
{ }^{40} \mathrm{~K} & \rightarrow{ }^{40} \mathrm{Ca}+\mathrm{e}^{+}+\bar{\nu}_{\mathrm{e}}+1.311 \mathrm{MeV} \\
{ }^{40} \mathrm{~K}+\mathrm{e}^{-} & \rightarrow{ }^{40} \mathrm{Ar}+\nu_{\mathrm{e}}+1.505 \mathrm{MeV}
\end{aligned}
$$

(e.g., Dye, 2012). ${ }^{40} \mathrm{~K}$ decays to ${ }^{40} \mathrm{Ca} 89.3 \%$ of the time. The energy produced by these reactions (kinetic energies of alpha and beta particles, plus gamma rays produced by decays and electron-positron annihilations) is the radiogenic heating of Earth. The antineutrinos of the ${ }^{238} \mathrm{U}$ and ${ }^{232} \mathrm{Th}$ decay chains escape and do not heat Earth but are detectable by the KamLAND and Borexino neutrino observatories (Araki et al., 2005; Dye, 2012; Ludhova, 2012; Tolich, 2012). These experiments have shown that these isotopes account for $>5 \mathrm{TW}$ (probably $\sim 19 \mathrm{TW}$ ) of internal energy production, out of a total rate of $\sim 44 \mathrm{TW}$ (Araki et al., 2005; KamLAND Collaboration, 2011; Dye, 2012). For this study, we will assume a Th/U ratio of 3.9 (Anders and Ebihara, 1982; Anders and Grevesse, 1989), similar to chondritic, and a low, but observationally permitted, $\mathrm{K} / \mathrm{U}$ ratio of 12,000 (Arevalo et al., 2009), 40 times less than chondritic (Anders and Grevesse, 1989). Earth is enriched in U relative to chondrites but depleted in $\mathrm{K}$. The half-lives of the ${ }^{235} \mathrm{U},{ }^{238} \mathrm{U},{ }^{232} \mathrm{Th}$, and ${ }^{40} \mathrm{~K}$ species are $0.7,4.5,13.9$, and $1.4 \mathrm{Gyr}$, respectively. Therefore, $\mathrm{K}$ dominates early on, but Th dominates on long timescales.

Figure 6 shows the nominal evolution of Earth's surface energy flux over time due to radiogenic heating (assuming the energy is instantly transported to the surface), as well as several alternative scenarios. The "Earth" curve is the above model in which $19 \mathrm{TW}$ (8 TW each from $\mathrm{U}$ and Th, $3 \mathrm{TW}$ from ${ }^{40} \mathrm{~K}$ ) is generated today. The "Only Radiogenic" curve assumes that the entire $44 \mathrm{TW}$ is generated radiogenically, with the same elemental proportions as the "Earth" case. The "Chondritic Earth" case assumes Earth formed its current $\mathrm{U}$ inventory but with 40 times more ${ }^{40} \mathrm{~K}$ than the "Earth" case, and the "Twice Chondritic, Only Radiogenic" case has 80 times the ${ }^{40} \mathrm{~K}$ proportion and twice the U and Th proportion as the "Only Radiogenic" model. These latter curves are plausible for terrestrial exoplanets that formed beyond the snow line and migrated in during the protoplanetary disk phase (Carter-Bond et al., 2012) and hence might not be depleted in K. Note that, if Earth formed with a chondritic K/U ratio, it could have experienced Iolike heat fluxes at its surface for $\sim 1.5 \mathrm{Gyr}$.

In addition to radiogenic heating, tidal heating could be significant on terrestrial bodies in the HZ of low-mass stars. The tidal heating in the interior is a strong function of composition, as well as external factors such as the mass of the star and the orbit. We consider tidal heating as predicted

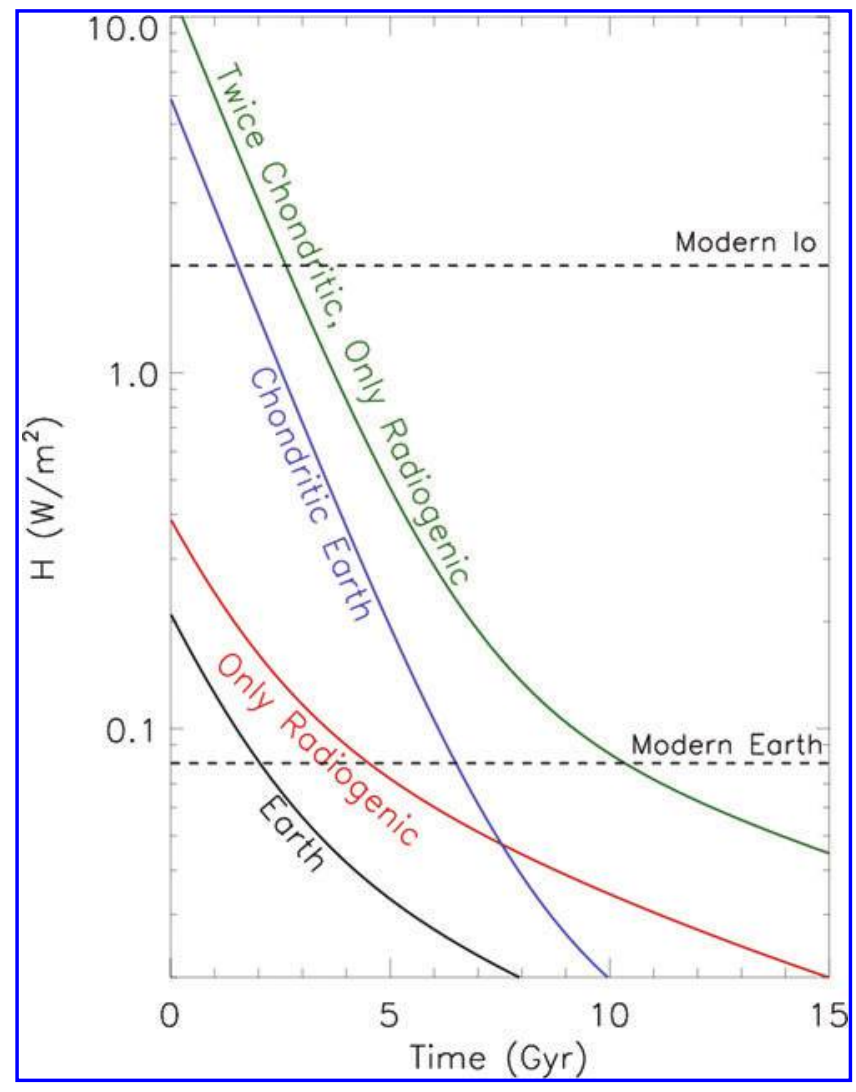

FIG. 6. Surface energy fluxes due to radiogenic heating, including comparisons to modern Earth and modern Io. Black curve: Our nominal model for Earth in which modern Earth produces $19 \mathrm{TW}$ of radiogenic power coming from $8 \mathrm{TW}$ each of $U$ and Th and $3 \mathrm{TW}$ from ${ }^{40} \mathrm{~K}$. Red curve: The total $44 \mathrm{TW}$ of internal power of Earth is generated by $\sim 18 \mathrm{TW}$ each of $\mathrm{U}$ and Th, and $\sim 7 \mathrm{TW}$ from ${ }^{40} \mathrm{~K}$. Blue curve: Same as the black curve but with 40 times the K, i.e., chondritic K/U ratio. Green curve: The power from Earth if all its current energy were radiogenic, but then scaled to twice the chondritic K/U ratio. Color images available online at www.liebertonline.com/ast 
by the "constant-time-lag model" (e.g., Hut, 1981; FerrazMello et al., 2008; Leconte et al., 2010) and compare tidal heating to radiogenic heating on a hypothetical terrestrial exoplanet. For the sake of brevity, we do not reproduce the model here but refer the reader to Appendix E of Barnes et al. (2013) for a complete description.

As a specific example, we consider an Earth-mass, Earthradius planet orbiting Barnard's Star with an initial eccentricity of 0.19 and a semimajor axis of $0.06 \mathrm{AU}$ so that it experiences approximately the same insolation as Earth. Such a planet would likely have formed at larger distances and migrated in, as accumulation of local material is unlikely to form such a "large" planet around such a small star (Raymond et al., 2007). We assume the planet has a tidal time lag of $640 \mathrm{~s}$, similar to the value for modern Earth (Lambeck, 1977), and allow the planet's orbit and, hence, tidal heating to evolve with time. We assume $90 \%$ of the tidal dissipation occurs in a putative ocean and only $10 \%$ in the rocky interior. In addition, we assume the planet has a "Twice Chondritic, Only Radiogenic" composition. Initially, the radiogenic heating dominates, primarily due to ${ }^{40} \mathrm{~K}$; then after about $5 \mathrm{Gyr}$, tides dominate, as shown in Fig. 7. While this planet would experience Io-like conditions for its first 12 Gyr, it would subsequently settle into a relatively stable situation during which its internal energy would remain with a factor of 10 of the modern Earth for the next 50 Gyr. As Barnard's Star is expected to survive for trillions of years, tidal heating could in principle sustain geochemical cycles like plate tectonics and the carbonate-silicate cycle far longer than radiogenic heating, especially if another planetary companion can maintain a nonzero eccentricity. A tidally heated exoplanet with a composition different from Earth's could easily have different material properties that affect tidal dissipation.

\subsection{Internal structure and dynamics of terrestrial exoplanets (Sang-Heon Shim)}

While there are differences in their compositions, possibly due to alteration of composition during planet formation and differentiation (Ringwood, 1977; Zindler and Hart, 1986), the abundances of nonvolatile elements in the solar photosphere, type I carbonaceous chondrites (CI chondrites), and Earth's upper mantle (Taylor and McLennan, 1985) are very similar. This gives hope that stellar elemental abundances may provide us with important constraints on the bulk chemical compositions of planets around stars. We explore two examples that show how variations in some key elemental ratios may affect the composition, structure, and dynamics of planetary interiors. Even for our own planet, many key issues are still under debate, such as the chemical composition of the deep mantle and the core and the phase relations and the physical properties of key constituents (minerals and melts). Therefore, we will limit our discussions to changes in major mineralogy with simplified compositions and their possible impacts on just a few properties of planetary interiors.

3.4.1. C/O ratio. Some stars have much higher $\mathrm{C} / \mathrm{O}$ than that of the Sun (Bond et al., 2010a, 2010b), and the elevated $\mathrm{C} / \mathrm{O}$ may result in carbide mineralogy, instead of silicate mineralogy, during nebular condensation (Larimer, 1975). Stars with high $\mathrm{C} / \mathrm{O}$ could host carbide planets (silicon car-

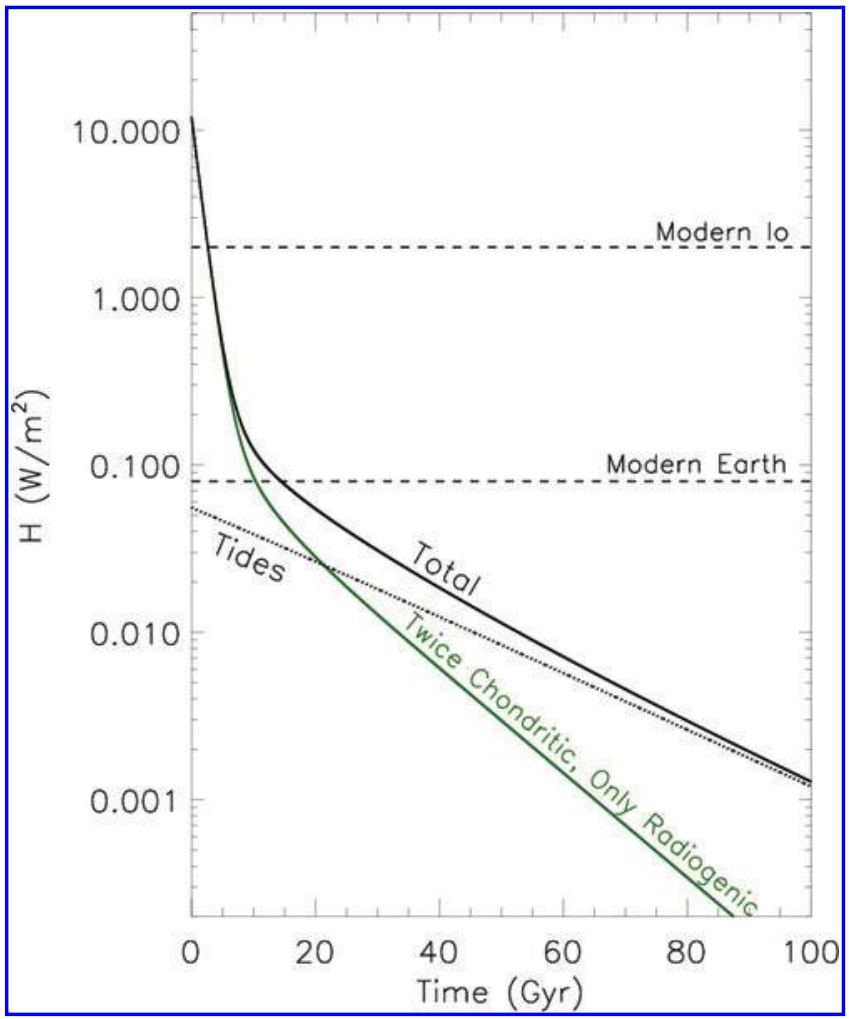

FIG. 7. Comparison of tidal and radiogenic heating on a hypothetical Earth-mass, Earth-radius planet in the HZ of Barnard's Star, initially with a semimajor axis of $0.06 \mathrm{AU}$ and eccentricity of 0.19 . The planet's radiogenic inventory is the same as the "Twice Chondritic, Only Radiogenic" curve from Fig. 6. The tidal heating of the rocky interior is generated by the slowly damping semimajor axis and shown by the dotted curve. The green curve is the same as in Fig. 6. The solid black curve is the total heating flux. Despite the orbital evolution, the planet remains in the insolation HZ for the full $100 \mathrm{Gyr}$ shown. Color images available online at www.liebertonline.com/ast

bides and even elemental carbon in the mantle and iron carbides in the core). Some key thermal and rheological properties of $\mathrm{SiC}$ (moissanite) and $\mathrm{SiO}_{2}$ (quartz) are compared in Table 2. It is important to note that Earth's mantle consists mostly of magnesium silicates, not pure silica. Furthermore, high pressure in the planet's interior can change the physical properties of minerals. While extensive studies have been made of the properties of silica and silicates at high pressure-temperature conditions found in planetary interiors (Hemley, 1998, and references therein), much less is known for the properties of $\mathrm{SiC}$ at high pressure and temperature. Therefore, the comparison of the properties at 1 bar and $300 \mathrm{~K}$ discussed below is to highlight the fundamental differences in physical properties between silicates and carbides.

Silicon carbide polymorphs have very dense crystal structures even at 1 bar, while silica polymorphs have open crystal structures at the same conditions, resulting in fairly large contrast between them at 1 bar. With compression, $\mathrm{SiO}_{2}$ undergoes a series of polymorphic phase transitions to denser structures: coesite to stishovite at $\sim 9 \mathrm{GPa}$ (Stishov and Popova, 1961) and stishovite to seifertite at $\sim 120 \mathrm{GPa}$ (Grocholski et al., 2013). The density of seifertite at 1 bar is 
Table 2. Comparison of Some Physical Properties OF $\mathrm{SIO}_{2}$ AND SiC at 1 BAR AND $300 \mathrm{~K}$

\begin{tabular}{lcl}
\hline Physical properties & $\mathrm{SiO}_{2}$ & $\mathrm{SiC}$ \\
\hline $\begin{array}{l}\text { Density }\left(\mathrm{g} \mathrm{cm}^{-3}\right) \\
\text { Thermal expansion parameter }\end{array}$ & 2.66 & 3.21 \\
$\left(10^{-6} \mathrm{~K}^{-1}\right)$ & 12.3 & 4.0 \\
Thermal conductivity $\left(\mathrm{W} \mathrm{m}^{-1} \mathrm{~K}^{-1}\right)$ & 1.3 & 500 \\
\hline
\end{tabular}

$4.355 \pm 0.003 \mathrm{~g} \mathrm{~cm}^{-3}$, (compared to $2.93 \mathrm{~g} \mathrm{~cm}^{-3}$ for coesite) demonstrating profound impact of pressure (Grocholski et al., 2013). In contrast, $\mathrm{SiC}$ does not appear to have a major phase transition up to $90 \mathrm{GPa}$ (Yoshida et al., 1993).

More drastic contrasts can be found as well. SiC has a factor of 3 smaller thermal expansivity and a factor of 400 greater thermal conductivity. Of course, it is uncertain whether the magnitudes of these differences will remain the same at higher pressures. Nevertheless, this comparison shows that $\mathrm{SiC}$ may not gain buoyancy as much as silicates do by heating, and heat will be transported more effectively through conduction in $\mathrm{SiC}$ than in silicates, suggesting thermal conduction will be much more important as a heat transport process in carbide mantles than in Earth's silicate mantle, where convection is dominant (Davies, 1999). Because surface tectonics are closely related to internal processes, at least for Earth (through mantle convection), the possible dominance of thermal conduction over convection could result in significantly different surface processes in carbide planets, perhaps a lack of plate tectonics and/or lack of mixing between the mantle and surface.

It is important to point out that there remain important questions for the identification of carbide planets. The existing report on the identification of carbide planets (Madhusudhan et al., 2012) relies on knowledge of the thermal equations of state of $\mathrm{SiC}$ and diamond at high pressures, but the thermal equation of state of $\mathrm{SiC}$ has been estimated based on low-pressure spectroscopic measurements (Aleksandrov et al., 1989; Seager et al., 2007). Furthermore, the EOS does not take into account a phase transition in $\mathrm{SiC}$ reported at $90 \mathrm{GPa}$, which may increase density drastically ( 10-20\%) (Yoshida et al., 1993). For reliable identification of carbide planets, it therefore is urgent to establish a robust EOS of $\mathrm{SiC}$ at high pressure and temperature, including the effects of densification through the phase transition.

Recently, Sen et al. (2013) suggested substitution of C for $\mathrm{O}$ in $\mathrm{SiC}$ may be far more energetically possible at high pressures than substitution of $\mathrm{C}$ for $\mathrm{Si}$. If this indeed happens at high pressure and forms $\mathrm{SiO}_{x} \mathrm{C}_{4-x}$ type materials for carbon-rich planets, the different properties of such materials will have profound impact on internal dynamics and evolution of the planets. Yet it should be further examined if such materials can be actually stabilized under possibly very reducing conditions of carbon-rich planets.

3.4.2. $\mathrm{Mg} / \mathrm{Si}$ ratio. Earth's upper mantle is composed mainly of olivine, $(\mathrm{Mg}, \mathrm{Fe})_{2} \mathrm{SiO}_{4}$, and pyroxene, $(\mathrm{Mg}, \mathrm{Fe}$,$\mathrm{Ca})(\mathrm{Al}, \mathrm{Si}) \mathrm{O}_{3}$ with $\mathrm{Mg} / \mathrm{Si}=1.2-1.3$ (Ringwood, 1975; McDonough and Sun, 1995). With an increase in depth or pressure, these minerals undergo polymorphic phase transitions. Although this interpretation remains controversial, the $\mathrm{Mg} / \mathrm{Si}$ ratio may remain the same or similar throughout Earth's mantle, because the boundary between the upper mantle and the lower mantle of Earth can be explained by a polymorphic phase transition, the post-spinel transition (Ringwood, 1970; Ito and Takahashi, 1989; Shim et al., 2001).

Significant variations in $\mathrm{Mg} / \mathrm{Si}$ among different stars have been documented (Bond et al., 2010a, 2010b), ranging from 0.8 to 2 (see also Subsection 2.2), and this variation will result in significant changes in the mineralogy of the mantles in Earth-like exoplanets. For example, compared with Earth's mantle, a decrease in $\mathrm{Mg} / \mathrm{Si}$ will increase the amount of pyroxene and its high-pressure polymorphs and decrease the amount of olivine and its polymorphs. It is even possible to generate free silica $\left(\mathrm{SiO}_{2}\right)$, if $\mathrm{Mg} / \mathrm{Si}$ is sufficiently lower than 1, as shown for basaltic compositions (Hirose et al., 2005; Ricolleau et al., 2008). Free silica is not expected to exist in Earth's pyrolitic mantle. On the other hand, an increase in $\mathrm{Mg} / \mathrm{Si}$ to $\geq 2$ will yield upper mantles almost entirely made of olivine, and it is even possible that ferropericlase, $(\mathrm{Mg}, \mathrm{Fe}) \mathrm{O}$, may exist from very shallow depths. In Earth's mantle, ferropericlase exists only in the lower mantle where olivine breaks down to perovskite, $(\mathrm{Mg}, \mathrm{Fe})(\mathrm{Al}, \mathrm{Si}) \mathrm{O}_{3}$, and ferropericlase, $(\mathrm{Mg}, \mathrm{Fe}) \mathrm{O}$.

This may have profound impacts on mantle dynamics. For example, ferropericlase is known to have much smaller viscosity than other minerals in Earth's lower mantle, which is made of $70 \%(\mathrm{Mg}, \mathrm{Fe})(\mathrm{Al}, \mathrm{Si}) \mathrm{O}_{3}$ perovskite, $25 \%$ ferropericlase, and $5 \% \mathrm{CaSiO}_{3}$ perovskite. If the amount of ferropericlase increases to a level where ferropericlase grains are mechanically interconnected, the viscosity of the lower mantle may decrease by 2 orders of magnitude (Ammann et al., 2011). Such a significant decrease in viscosity would result in much more vigorous thermal convection in the mantle.

The change in $\mathrm{Mg} / \mathrm{Si}$ will also affect many other processes in the mantles of silicate planets, such as melting relations and phase transitions. For example, melting behavior has a profound impact on planet differentiation. Therefore, early evolution of silicate planets with different $\mathrm{Mg} / \mathrm{Si}$ may result in very different internal structures. Melting behaviors also affect near-surface processes. They will affect formation of crust and lithosphere, types of volcanism, and possibly surface dynamics and tectonics. Also, it is important to mention that $\mathrm{C} / \mathrm{O}$ may be still very important for understanding silicate planets as well, because it controls the redox conditions, which may affect the size and contents of light elements in the core (and therefore physical and chemical conditions), which, for example, may affect the planetary magnetic fields.

\section{Sources of Abundance Variations}

Having established that there are considerable variations in the abundances of individual elements in stars, and most likely planets, it is worth considering the possible sources of these variations. The elemental abundances of a stellar system depend primarily on the composition of the molecular cloud from which it forms, which is primarily set by overall galactic chemical evolution, or GCE (e.g., Tinsley, 1980; Timmes et al., 1995; Schönrich and Binney, 2009). A molecular cloud may differ in its chemical composition from other clouds, if it were to inherit its elements in different proportions from various sources.

There are six or seven major sites of nucleosynthesis in the Galaxy. One nucleosynthesis source is spallation, or 
modification of a nucleus through a collision with a highenergy cosmic ray. This is a significant production channel for a few isotopes of $\mathrm{Li}, \mathrm{Be}$, and $\mathrm{B}$. In general, it is not a major source of elements (Arnett, 1996).

Much more important are stellar nucleosynthesis sources involving stars with companions. Novae, thermonuclear explosions on the surface of white dwarfs accreting matter from a companion, create products of high-temperature hydrogen burning, including isotopes of $\mathrm{Na}, \mathrm{Mg}$, and $\mathrm{Al}$. Type Ia supernovae (SNeIa) involve the thermonuclear explosion and complete destruction of a white dwarf interacting with a companion. SNeIa produce alpha elements, so called because they can be produced by successive addition of alpha particles to atomic nuclei (C, O, Ne, Mg, Si, S, Ar, $\mathrm{Ca}, \mathrm{Ti}, \mathrm{Cr}, \mathrm{Mn}, \mathrm{Fe}$, and $\mathrm{Ni}$ ). SNeIa are the sources of more than half the iron in the galactic disk and produce significant amounts of $\mathrm{Si}$ and $\mathrm{Ca}$. Neutron star-neutron star mergers are predicted to eject $\sim 10^{-3} M_{\odot}$ of very high atomic number $r$-process (rapid neutron capture process) material (e.g., Goriely et al., 2011). All these examples create elements only many hundreds of millions of years after the progenitor stars form.

Single stars also contribute significantly. In low- to intermediate-mass $\left(<8 M_{\odot}\right)$ stars, deep convection dredges up material processed by nuclear burning during the stars' lifetimes, typically after hundreds of millions of years. This is the dominant source of $\mathrm{C}$ in the universe as well as $s$-process (slow neutron capture process) elements, roughly half the species between the iron peak and ${ }^{209} \mathrm{Bi}$. Stars above $\sim 25$ $M_{\odot}$, depending on metallicity, lose their entire hydrogen envelopes to eruptive phases as luminous blue variables and continue losing substantial mass as Wolf-Rayet stars, which are essentially bare stellar cores. Wolf-Rayet winds may contain products of hydrogen burning ( $\mathrm{He}$ with modified abundances of $\mathrm{Na}, \mathrm{Mg}, \mathrm{Al}$ ), partial He burning (high $\mathrm{C}$ abundance), or complete He burning (O-dominated with modified Ne abundances). Finally, stars above $\sim 8 M_{\odot}$ can explode as core collapse supernovae (CCSNe), which are the primary production site of $\mathrm{O}, \mathrm{Si}$, and a significant fraction of Fe. CCSNe eject many isotopes of elements with both even and odd proton numbers, for example $\mathrm{P}$ (unlike SNeIa, which produce mainly even- $Z$ elements). CCSNe also produce $r$ process elements, which are the remainder of the nuclei beyond the iron peak, including all nuclei heavier than ${ }^{209} \mathrm{Bi}$. Core collapse supernovae take place from a few to tens of millions of years after the formation of the progenitor star.

It is possible that stars within a cloud may differ chemically, if parts of the cloud can be contaminated by the products of stellar nucleosynthesis during the few tens of millions of years during which it is forming stars. The most natural candidates for such self-enrichment are Wolf-Rayet winds and core-collapse supernovae, since their progenitors live only $\sim 3$ to $20 \mathrm{Myr}$ before ejecting elements. Strong evidence exists for this process from isotopic analyses of meteorites in our Solar System, which show that it contained short-lived radionuclides such as ${ }^{41} \mathrm{Ca}\left(t_{1 / 2}=0.15 \mathrm{Myr}\right),{ }^{26} \mathrm{Al}\left(t_{1 / 2}=\right.$ $0.71 \mathrm{Myr})$, and ${ }^{60} \mathrm{Fe}\left(t_{1 / 2}=2.3 \mathrm{Myr}\right)$ at levels well above those expected from GCE (Wadhwa et al., 2007; Huss et al., 2009). This points to an injection of supernova material of $\sim 10^{-4}$ of the mass in the protoplanetary disk, less than a few $\times 10^{5}$ years before the formation of the first solids (Wadhwa et al., 2007). Stable isotope anomalies in meteorites paint a similar picture. Comparing the oxygen isotope ratios of the Solar System with those of nearby molecular clouds, Young et al. (2011) argued that $\sim 1 \%$ of the Solar System's oxygen was injected into the Sun's cloud after its composition was determined by GCE.

Even after a stellar system forms, elements can be mixed and redistributed within the star's protoplanetary disk. Planets forming at different places within the same disk may have very different abundances, especially of volatile species like $\mathrm{H}_{2} \mathrm{O}$ and $\mathrm{S}$.

\subsection{Injection of supernova material into forming stellar systems (Evan Scannapieco, Steven Desch)}

How supernova material could be injected into a forming solar system has been an outstanding issue for over 50 years (Cameron, 1962). Previous studies have investigated how a stable molecular cloud core could be induced to collapse as supernova material was injected into it (Cameron and Truran, 1977; Boss, 1995; Foster and Boss, 1995; Boss and Vanhala, 2000), how a star-forming complex could be contaminated by waves of propagating star formation (Gounelle et al., 2009), and how supernova material could be injected into an already-formed protoplanetary disk (Chevalier, 2000; Ouellette et al., 2007, 2010).

More recently, Pan et al. (2012) studied how a single supernova can inject material into a nearby molecular cloud. Because of their intense UV emission, very massive stars ( $>30 M_{\odot}$ ) alter the birth environment of neighboring stars. The ionization fronts they launch move slowest through the dense regions in which stars form and could also trigger further star formation at these boundaries (see review by Hester and Desch, 2005). Massive stars explode as supernovae after $\sim 4 \mathrm{Myr}$ (Hansen et al., 2004) and can potentially contaminate the protostars at the edges of these H II regions, which are typically a few parsecs away at that time.

One of the findings by Pan et al. (2012) was that isotropically exploding supernova ejecta do not mix into molecular clouds; but clumpy ejecta, as seen in the Cassiopeia A supernova remnant (Fesen et al., 2001; Hwang et al., 2004), do mix. These clumps resemble those that are robustly predicted to form in supernovae (e.g., Kifonidis et al., 2003; Ellinger et al., 2012) and inferred to be the sites of supernova dust condensation (Fedkin et al., 2010). Ouellette et al. (2010), Ellinger et al. (2012), and Pan et al. (2012) modeled these ejecta clumps (which arise interior to the $\mathrm{H}$ and He-burning shells) as having masses $\sim 10^{-4} M_{\odot}$, diameters $\sim 10^{16} \mathrm{~cm}$, ejection velocities between 2000 and $6000 \mathrm{~km} \mathrm{~s}^{-1}$, and densities $\sim 4 \times 10^{-19} \mathrm{~g} \mathrm{~cm}^{-3}$. These densities are similar to those of a typical molecular cloud, allowing the clumps to penetrate and mix into it. It is essential to understand that such clumps are extremely heterogeneous in composition. The makeup of each clump reflects the abundances in the particular part of the star/explosion where it formed (Young et al., 2009; Ellinger et al., 2012). Smallscale chemical mixing of clumpy supernova material is minimal. For example, the composition of one supernova bullet that formed from the O-rich shell of the star will have a completely different composition from one formed out of the C- and Ne-burning shell of the same object.

The interaction between such a clump and a nearby molecular cloud, as modeled by Pan et al. (2012) using the 
FLASH hydrodynamics code (Fryxell et al., 2000), is shown in Fig. 8. Initially, the clump (a small dot on the scale of the figure) is just outside the molecular cloud at $x=0$ and moving to the right at $2000 \mathrm{~km} \mathrm{~s}^{-1}$. At $t=5 \mathrm{kyr}$, it has carved out a channel $0.4 \mathrm{pc}$ long and deposited ejecta material along it, and some material has escaped back into the $\mathrm{H}$ II region to the left. At $t=30 \mathrm{kyr}$, the clump has stalled at a depth of $0.5 \mathrm{pc}$, and $86 \%$ of the clump mass remains in the cloud.

Subsequent turbulent mixing will distribute the ejecta laterally, so that all the gas within $0.5 \mathrm{pc}$ of the edge of the $\mathrm{H}$ II region will be contaminated. The roughly $2 M_{\odot}$ of supernova ejecta will thus mix with about $4700 M_{\odot}$ (assuming the ionization fronts are $4 \mathrm{pc}$ from the massive star). All stars in the act of forming at the time of the supernova will be contaminated with ejecta at a level $\sim 10^{-4}$ to $10^{-3}$, which is sufficient to explain the abundance of ${ }^{26} \mathrm{Al}$ and potentially other short-lived radionuclides (Pan et al., 2012). Because the clumps are $\sim 100$ times more metal-rich than a solar composition gas, they can alter the abundance of oxygen or other elements by 1-10\% as well (Pan et al., 2012), possibly explaining the isotopic shifts inferred by Young et al. (2011). Based on the rates of triggered star formation estimated by Snider et al. (2009), Pan et al. (2012) estimated that 10-20\% of all Sun-like stars may experience such $1-10 \%$ shifts in elemental abundances, especially in primary isotopes like ${ }^{12} \mathrm{C}$ and ${ }^{16} \mathrm{O}$.

\subsection{Snow lines in externally photoevaporated disks (Steven Desch)}

Numerous models predict efficient radial transport of elements in a protoplanetary disk, and redistribution of volatiles in particular. A famous example is the case of water, which can diffuse as vapor out of the inner parts of disks,

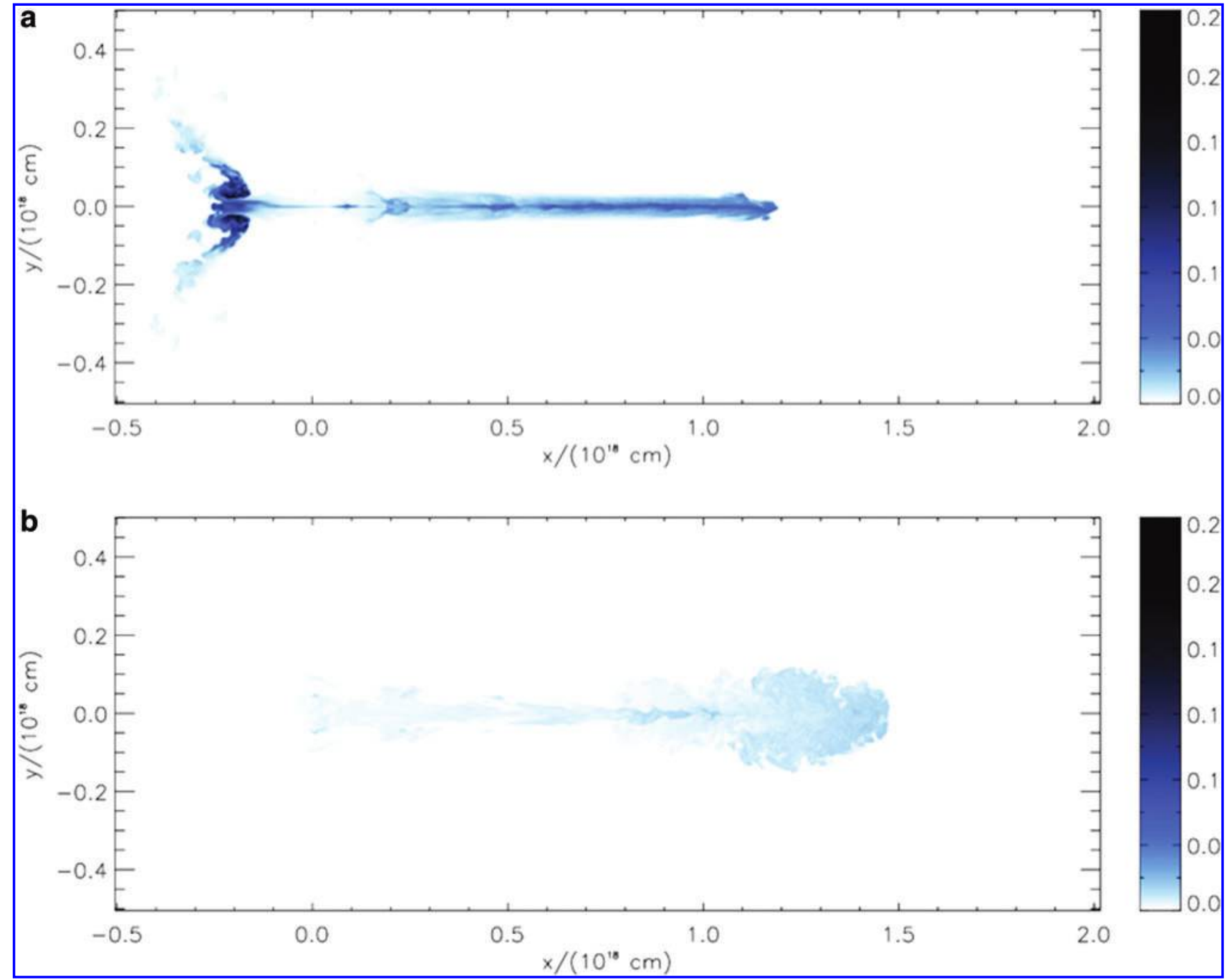

FIG. 8. Concentration of supernova material in a molecular cloud, following injection of a "bullet" of supernova ejecta. The ejecta clump (see text) enters the molecular cloud from the H II region to the left, at $2000 \mathrm{~km} \mathrm{~s}^{-1}$, striking the boundary of the cloud at $x=0$. Color denotes the mass fraction of ejecta at each location. (Top) The ejecta 5 kyr after impact, showing deposition of ejecta along a narrow channel in the cloud, as well as high concentrations in a plume escaping through the channel into the H II region. (Bottom) The ejecta $30 \mathrm{kyr}$ after impact, when its forward momentum is arrested, showing moderate concentrations along the channel, to a depth of $0.5 \mathrm{pc}$. Eighty-six percent of the ejecta remain in the molecular cloud. Color images available online at www.liebertonline.com/ast 
condensing and remaining trapped in the colder outer portions of the disk (Stevenson and Lunine, 1988; Cyr et al., 1998). As ice particles grow, they may radially drift inward with respect to the gas, bringing water back into the inner nebula. A steady-state balance, with some water vapor in the inner disk and an enhancement of ice in the outer disk, may subsequently be established (Cuzzi and Zahnle, 2004; Ciesla and Cuzzi, 2006). The boundary between these two regionstermed the "snow line" - is taken to be where the temperature $T \approx 170 \mathrm{~K}$, the condensation temperature of water ice at nebular pressures (e.g., Lodders, 2003). Large enhancements of ice, an order-of-magnitude increase above normal cosmochemical abundances, are possible beyond the snow line, while water can be virtually absent inside the snow line.

A representative calculation by Desch (2012) is depicted in Fig. 9, which shows the ratio of ice column density to rock column density as a function of heliocentric distance, at $0.5 \mathrm{Myr}$ increments, in a viscously evolving protoplanetary disk. In this toy-model calculation, the disk evolves according to the standard equations (Lynden-Bell and Pringle, 1974; Hartmann et al., 1998), and the temperature of the disk depends on the rate of mass accretion through the disk, $\mathrm{d} M / \mathrm{d} t$. As $\mathrm{d} M / \mathrm{d} t$ decreases over time, the temperature drops everywhere in the disk, and the distance where $T=170 \mathrm{~K}$ moves inward. At each point, water can exist as vapor or ice, and diffusion of vapor and inward migration of large ice particles relative to the gas are accounted for in a parameterized fashion, as are the diffusion of dust and inward migration of large rocky particles. As predicted, the ice/rock ratio is enhanced over average solar nebula values beyond the $T=170 \mathrm{~K}$ line and essentially vanishes inside it. As the

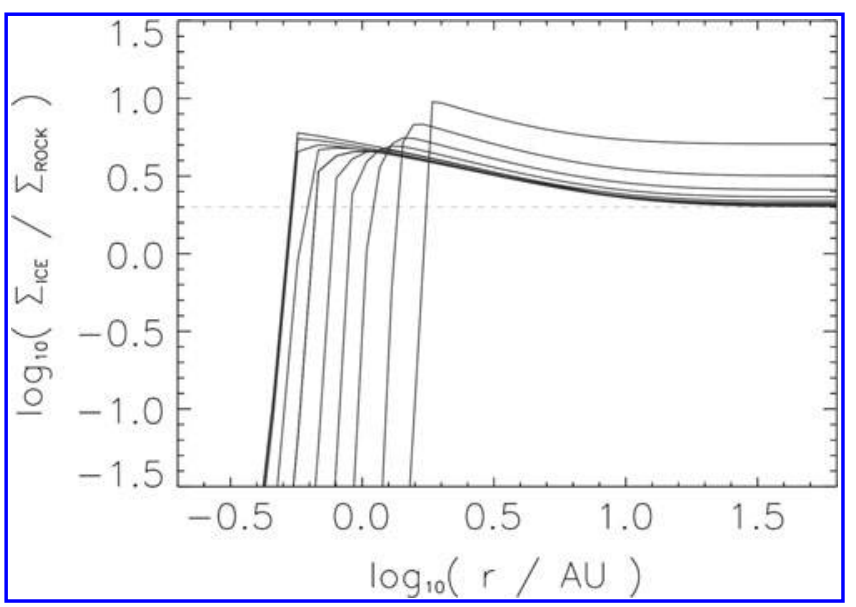

FIG. 9. Ratio of surface density of ice to surface density of rock, as a function of heliocentric distance, at various times in the evolution of a protoplanetary disk. Dashed line denotes the cosmochemical ratio. From right to left, the times depicted are disk ages of 0.5-5.0 Myr, incremented by $0.5 \mathrm{Myr}$. Water is in vapor form, not ice, interior to the radius where $T=170 \mathrm{~K}$ (the snow line); ice is enhanced above solar abundances (dashed line) beyond the snow line, as vapor diffusing outward is cold-trapped there. Outward diffusion of vapor is balanced by inward migration of large icy solids. Over time, as mass accretion decreases, the temperature in the disk decreases and the snow line moves inward. nebula cools, the region of enhanced ice/rock ratio, the snow line, moves inward as well.

Such snow line models are invoked to explain the distribution of water within our solar system. S-type asteroids, associated with relatively dry $(<0.1 \mathrm{wt} \%$ water $)$ ordinary chondrites, are found predominantly inside 2.7 AU from the Sun, while C-type asteroids, associated with relatively wet ( $>10 \mathrm{wt} \%$ structurally bound water) carbonaceous chondrites, are found outside 2.7 AU. As it happens, the blackbody temperature of an isolated grain at 2.7 AU, exposed to the present-day Sun, is $170 \mathrm{~K}$. Thus $2.7 \mathrm{AU}$ is taken to be the snow line in the solar nebula. But as can be seen in Fig. 9, temperatures are cold enough to condense ice well inside 2.7 AU. Ordinary and carbonaceous chondrites formed while the disk gas was still present, at an approximate disk age of 3 Myr. Because disks are dusty and absorb sunlight obliquely, temperatures in the disk are far colder than the blackbody temperatures at the same locations. Viscous dissipation can add accretional heating as mass accretes through the disk at a rate $\mathrm{d} M / \mathrm{d} t$ through each annulus, but the observed mass accretion rates in disks $3 \mathrm{Myr}$ old are relatively low, $\mathrm{d} M / \mathrm{d} t<10^{-8} M_{\odot} \mathrm{yr}^{-1}$ (Gullbring et al., 1998). Under these conditions, models of disk temperatures almost universally predict $T<170 \mathrm{~K}$ no farther from the Sun than $2 \mathrm{AU}$ (Lecar et al., 2006), and more detailed models that account for the vertical structure of the viscous dissipation would put the snow line inside $1 \mathrm{AU}$ (Lesniak and Desch, 2011). If a snow line existed in the solar nebula, it was probably closer to $1 \mathrm{AU}$, certainly well inside $2.7 \mathrm{AU}$. This raises the question of why the inner Solar System is so dry. Why did Earth and Mars not accrete as much ice as rock?

A possible solution to the problem, proposed by Desch (2012), is photoevaporation of the Sun's protoplanetary disk by the FUV radiation from a nearby massive $\mathrm{O}$ star. Desch (2007) demonstrated that externally photoevaporated disks can experience steady-state net outward transport of mass from their inner regions to the disk edge being photoevaporated (typically at $50 \mathrm{AU}$ for a disk several parsecs from an O6 star; Adams et al., 2004). Desch (2012) hypothesized that this outward mass flux could prevent the inward drift of icy particles that balances the outward diffusion of water vapor across the snow line. Figure 10 depicts the same disk modeled in Fig. 9, only this time with external photoevaporation from an 06 star 2 pc away included. Vapor diffuses out of the inner solar nebula but is never replenished, and the inner Solar System can lose essentially all its water ice despite being more than cold enough for ice to condense.

\section{Implications and Future Directions}

Variations in element abundances among stars have consequences for the habitability of the planets orbiting them in ways that we are only beginning to discern but that promise the birth of entirely new multidisciplinary fields.

Historically, astrophysics and biology have intersected only at the surface of a planet, but it seems increasingly likely that the geochemistry and geophysics of planetary interiorswhich ultimately shape surface environments-are also strongly affected by abundance variations of the magnitude that is observed among nominally Sun-like stars. It is possible to envision vast new research frontiers opening up as mineral physicists, petrologists, and geophysicists begin to conduct 


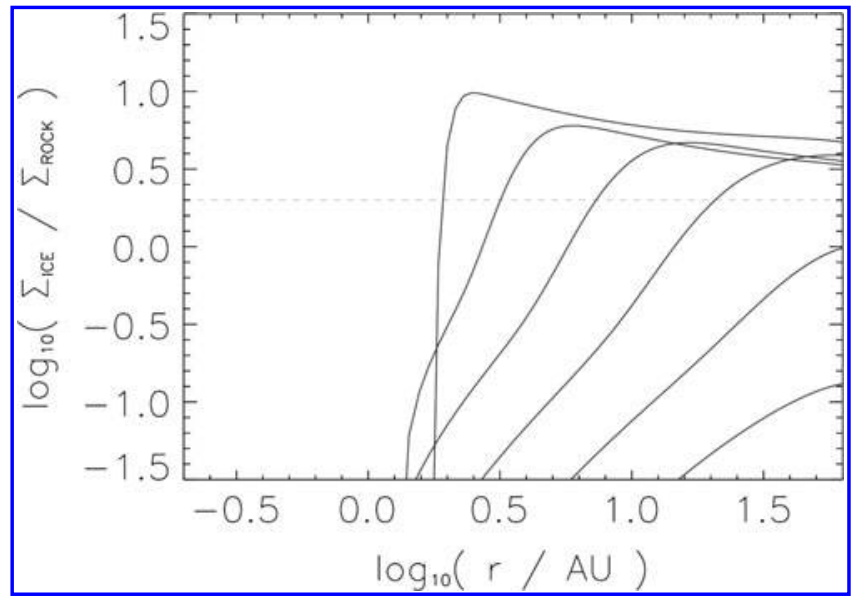

FIG. 10. Same as Fig. 9, except for a protoplanetary disk that is experiencing external photoevaporation at its outer edge (around $50 \mathrm{AU}$ ). From left to right the curves depict disk ages of $0.5-2.5 \mathrm{Myr}$, incremented every $0.5 \mathrm{Myr}$. As the disk evolves, external photoevaporation causes the net flux of matter to be outward, which prevents the inward migration of solids that would replenish the inner Solar System. In only $\sim 10^{6}$ years the inner solar nebula is essentially devoid of ice.

experiments and construct computational models that deviate from terrestrial compositions, taking astrophysical data as inputs and providing as outputs geophysical and geochemical constraints that will be crucial to predicting surface habitability. We propose the term "astrobiological stoichiometry" for the study of chemical compositions and their effects on star and planet formation, and planetary interior, surface, and atmospheric processes that influence the habitability of extrasolar planetary systems.

While the current research trends are exciting and tantalizing, there is a danger of overgeneralization and overapplication of early results. There are critical areas where fundamental astrophysical work is needed before emerging notions of planetary habitability can mature.

First, and most obvious, is the observational determination of chemical compositions of both stars and planets. Determining accurate compositions is not straightforward, even for the most easily available targets, the host stars of extrasolar planets. Researchers in this field must perform detailed comparisons of their methods to identify the sources of discrepancies. That information must be used to agree upon a set of best practices for abundance determination, including optimal sets of atomic constants, line lists, line flux measurement strategies, and stellar parameter determination. Independent verification of stellar parameters by methods such as asteroseismology should be used where possible. Finally, it is essential to determine the abundances of as many elements as possible for any interesting host star. The Fe abundance alone is not sufficient even to accurately describe the host star's evolution.

Second, greater progress must be made in deriving abundances of planets. For now observations are restricted to absorption or reflection spectra from jovian planets that are transiting or directly observable. The few existing observations to date already confirm that planets differ compositionally from their stars. These differences could arise from disk processes during planet formation such as volatile transport and loss from the protoplanetary disk; con- densation of minerals; or radial mixing of gas, dust, and planetesimals. Chemical abundances can be changed as a planet accretes, and elements can partition differently within the planet itself. This is illustrated by the apparent lack of oxygen in Jupiter's atmosphere as probed by the Galileo probe, which Juno may confirm to be a global depletion of oxygen from Jupiter's upper atmosphere, possibly due to dissolution of oxygen with neon into helium droplets. Terrestrial exoplanets so far cannot yield any direct composition information, only estimates of bulk density when their radii are known from transits and mass from orbital elements.

While observations of stars and planets improve, other observations can help constrain how stellar compositions translate into planetary abundances. Dust in young systems' debris disks left over from planet formation, produced by collisions between small parent bodies, represents material as it condensed out of a protoplanetary disk but before it was substantially processed in full-fledged planets. Near IR and mid-IR spectroscopy of such disks can be used to identify mineral species, giving insight into elemental ratios, potentially even as a function of distance from the star. A second source of dust, seen in a handful of systems, is impacts between large planetary bodies. Astromineralogical studies of these systems can provide insight into the mineral species that formed in high temperatures and pressures in planetary interiors.

A goal for the future is to model the creation of planets from an initial nebular composition to a planetary body, with the caveat that many steps along the way are stochastic. Dust condensation models exist for non-solar compositions and can be generalized further (Ebel, 2000, 2013). Disk models can compute how volatiles and minerals are transported through the disk and provide initial conditions for models of planetesimal and planet assembly. Migration of planets during and following assembly can be included. While the stochasticity of all these processes requires a large number of realizations of simulations, stellar compositions should be "mappable" onto planets in an average sense. After formation of a terrestrial planet, various planetary processes are open to exploration. Differentiation, thermal evolution, convection, mineralogy and rheology, and exchange between atmospheres and planetary surfaces through outgassing, erosion, and sequestration are all active fields of research. This set of problems will hopefully serve as an impetus to increase existing interaction between these fields and initiate new connections.

The fundamental purpose of astrobiology is to discover life on a world around another star. As our search has progressed, the options have multiplied: recent estimates are that $20 \%$ of Sun-like stars harbor a terrestrial planet at orbital distances that would yield Earth-like habitability. For the first time, astrobiology is faced with the problem of narrowing the parameters and creating a refined target list in the search for life. Astrobiological stoichiometry provides a robust strategy for hunting for life. As the field develops along the outlines explored here, we will be able to use readily observable stellar and planetary properties, especially chemical composition, and make predictions about the surface conditions on planets that are candidates for detailed follow-up in a next generation of missions searching for biosignatures. Compositional information is a key component 
in predicting the long-term climate evolution and surface geochemistry of extrasolar planets. Surface geochemistry, in turn, determines the total free energy available to an ecosystem and makes possible comparisons between the rates of abiotic chemical cycles and metabolic rates for hypothetical biological communities. These comparisons are vital to predicting the likelihood of a planet having detectable biosignatures. Chemical abundances are nearly as fundamental to habitability as stellar types and planetary orbits.

\section{Acknowledgments}

We thank the NASA Astrobiology Institute for supporting the research presented here and for making possible the Stellar Stoichiometry Workshop Without Walls, especially Estelle Dodson and Mike Toillon. We thank the many attendees who contributed scientifically to the workshop discussion.

A. Anbar, S. Desch, N. Hinkel, M. Pagano, M. Riner, A. Truitt, and P. Young gratefully acknowledge support from NASA's Follow the Elements team under cooperative agreement NNH08ZDA002C. R. Barnes acknowledges support by NASA's Virtual Planetary Laboratory lead team under cooperative agreement No. NNH05ZDA001C and NSF award AST-1108882. M. Pagano and P. Young acknowledge support from NSF award AST-0807567. S. Desch and N. Monga acknowledge support from NSF award AST-1108882. E. Scannapieco acknowledges support from NSF award AST1103608. R. Kopparapu gratefully acknowledges funding from the NASA Astrobiology Institute's Virtual Planetary Laboratory lead team, supported by NASA under cooperative agreement NNH05ZDA001C, and the Penn State Astrobiology Research Center.

\section{Author Disclosure Statement}

No competing financial interests exist.

\section{Abbreviations}

CCSNe, core collapse supernovae; EOS, equation of state; FUV, far ultraviolet; GCE, galactic chemical evolution; HZ, habitable zone; SNeIa, type Ia supernovae.

\section{References}

Abe, Y., Abe-Ouchi, A., Sleep, N.H., and Zahnle, K.J. (2011) Habitable zone limits for dry planets. Astrobiology 11:443-460.

Adams, F.C., Hollenbach, D., Laughlin, G., and Gorti, U. (2004) Photoevaporation of circumstellar disks due to external far-ultraviolet radiation in stellar aggregates. Astrophys $J$ 611:360-379.

Aleksandrov, I.V., Goncharov, A.F., Stishov, S.M., and Yakovenko, E.V. (1989) Equation of state and Raman scattering in cubic $\mathrm{BN}$ and $\mathrm{SiC}$ at high pressure. Journal of Experimental and Theoretical Physics Letters 50:127-131.

Ammann, M.W., Brodholt, J.P., and Dobson, D.P. (2011) Ferrous iron diffusion in ferro-periclase across the spin transition. Earth Planet Sci Lett 302:393-402.

Anders, E. and Ebihara, M. (1982) Solar-system abundances of the elements. Geochim Cosmochim Acta 46:2363-2380.

Anders, E. and Grevesse, N. (1989) Abundances of the elements: meteoritic and solar. Geochim Cosmochim Acta 53:197-214.

Araki, T., Enomoto, S., Furuno, K., Gando, Y., Ichimura, K., Ikeda, H., Inoue, K., Kishimoto, Y., Koga, M., Koseki, Y., Maeda, T., Mitsui, T., Motoki, M., Nakajima, K., Ogawa, H.,
Ogawa, M., Owada, K., Ricol, J.-S., Shimizu, I., Shirai, J., Suekane, F., Suzuki, A., Tada, K., Takeuchi, S., Tamae, K., Tsuda, Y., Watanabe, H., Busenitz, J., Classen, T., Djurcic, Z., Keefer, G., Leonard, D., Piepke, A., Yakushev, E., Berger, B.E., Chan, Y.D., Decowski, M.P., Dwyer, D.A., Freedman, S.J., Fujikawa, B.K., Goldman, J., Gray, F., Heeger, K.M., Hsu, L., Lesko, K.T., Luk, K.-B., Murayama, H., O'Donnell, T., Poon, A.W.P., Steiner, H.M., Winslow, L.A., Mauger, C., McKeown, R.D., Vogel, P., Lane, C.E., Miletic, T., Guillian, G., Learned, J.G., Maricic, J., Matsuno, S., Pakvasa, S., Horton-Smith, G.A., Dazeley, S., Hatakeyama, S., Rojas, A., Svoboda, R., Dieterle, B.D., Detwiler, J., Gratta, G., Ishii, K., Tolich, N., Uchida, Y., Batygov, M., Bugg, W., Efremenko, Y., Kamyshkov, Y., Kozlov, A., Nakamura, Y., Karwowski, H.J., Markoff, D.M., Nakamura, K., Rohm, R.M., Tornow, W., Wendell, R., Chen, M.-J., Wang, Y.-F., and Piquemal, F. (2005) Experimental investigation of geologically produced antineutrinos with KamLAND. Nature 436:499-503.

Arevalo, R., McDonough, W.F., and Luong, M. (2009) The K/U ratio of the silicate Earth: insights into mantle composition, structure and thermal evolution. Earth Planet Sci Lett 278:361-369.

Arnett, D. (1996) Supernovae and Nucleosynthesis, Princeton University Press, Princeton, NJ.

Asplund, M., Grevesse, N., Sauval, A.J., and Scott, P. (2009) The chemical composition of the Sun. Annu Rev Astron Astrophys 47:481-522.

Atreya, S.K., Wong, M.H., Owen, T.C., Mahaffy, P.R., Niemann, H.B., de Pater, I., Drossart, P., and Encrenaz, Th. (1999) A comparison of the atmospheres of Jupiter and Saturn: deep atmospheric composition, cloud structure, vertical mixing, and origin. Planet Space Sci 47:1243-1262.

Bar-Nun, A., Herman, G., Laufer, D., and Rappaport, M.L. (1985) Trapping and release of gases by water ice and implications for icy bodies. Icarus 67:317-332.

Barnes, R., Mullins, K., Goldblatt, C., Meadows, V.S., Kasting, J.F., and Heller, R. (2013) Tidal Venuses: triggering a climate catastrophe via tidal heating. Astrobiology 13:225-250.

Bond, J.C., Tinney, C.G., Butler, R.P., Jones, H.R.A., Marcy, G.W., Penny, A.J., and Carter, B.D. (2006) The abundance distribution of stars with planets. Mon Not R Astron Soc 370:163-173.

Bond, J.C., Lauretta, D.S., Tinney, C.G., Butler, R.P., Marcy, G.W., Jones, H.R.A., Carter, B.D., O'Toole, S.J., and Bailey, J. (2008) Beyond the iron peak: $r$ - and $s$-process elemental abundances in stars with planets. Astrophys J 682:1234-1247.

Bond, J.C., Lauretta, D.S., and O'Brien, D.P. (2010a) Making the Earth: combining dynamics and chemistry in the Solar System. Icarus 205:321-337.

Bond, J.C., OBrien, D.P., and Lauretta, D.S. (2010b) The compositional diversity of extrasolar terrestrial planets. I. In situ simulations. Astrophys J 715:1050-1070.

Boss, A.P. (1995) Collapse and fragmentation of molecular cloud cores. 2. Collapse induced by stellar shock waves. Astrophys J 439:224-236.

Boss, A.P. and Vanhala, H.A.T. (2000) Triggering protostellar collapse, injection and disk formation. Space Sci Rev 92:13-22.

Burrows, A. and Sharp, C.M. (1999) Chemical equilibrium abundances in brown dwarf and extrasolar giant planet atmospheres. Astrophys J 512:843-863.

Caffau, E., Ludwig, H.G., Malherbe, J.M., Bonifacio, P., Steffen, M., and Monaco, L. (2013) The photospheric solar oxygen project. II. Non-concordance of the oxygen abundance derived from two forbidden lines. Astron Astrophys 554:126-134. 
Cameron, A.G.W. (1962) Formation of the Sun and planets. Icarus 1:13-69.

Cameron, A.G.W. and Truran, J.W. (1977) The supernova trigger for formation of the Solar System. Icarus 30:447-461.

Carter-Bond, J.C., O'Brien, D.P., and Raymond, S.N. (2012) The compositional diversity of extrasolar terrestrial planets. II. Migration simulations. Astrophys J 760, doi:10.1088/ 0004-637X/760/1/44.

Charbonneau, D., Brown, T.M., Noyes, R.W., and Gilliland, R.L. (2002) Detection of an extrasolar planet atmosphere. Astrophys J 568:377-384.

Charbonneau, D., Berta, Z.K., Irwin, J., Burke, C.J., Nutzman, P., Buchhave, L.A., Lovis, C., Bonfils, X., Latham, D.W., Udry, S., Murray-Clay, R.A., Holman, M.J., Falco, E.E., Winn, J.N., Queloz, D., Pepe, F., Mayor, M., Delfosse, X., and Forveille, T. (2009) A super-Earth transiting a nearby low-mass star. Nature 462:891-894.

Chen, B., Li, J., and Hauck, S.A. (2007) Effect of pressure on the melting behavior of the $\mathrm{Fe}-\mathrm{S}$ system at moderate pressures: insight into the state of Mercury's core [abstract \#DI31A-0257]. In American Geophysical Union, Fall Meeting 2007, American Geophysical Union, Washington, DC.

Chevalier, R.A. (2000) Young circumstellar disks near evolved massive stars and supernovae. Astrophys J 538:L151-L154.

Ciesla, F.J. and Cuzzi, J.N. (2006) The evolution of the water distribution in a viscous protoplanetary disk. Icarus 181:178-204.

Clementi, E., Matcha, R., and Veillard, A. (1967) Simple basis set for molecular wavefunctions containing third-row atoms. J Chem Phys 47:1865-1866.

Cuzzi, J.N. and Zahnle, K.J. (2004) Material enhancement in protoplanetary nebulae by particle drift through evaporation fronts. Astrophys J 614:490-496.

Cyr, K.E., Sears, W.D., and Lunine, J.I. (1998) Distribution and evolution of water ice in the solar nebula: implications for Solar System body formation. Icarus 135:537-548.

Davies, G.F. (1999) Dynamic Earth: Plates, Plumes, and Mantle Convection, Cambridge University Press, Cambridge, UK.

De Silva, G.M., Sneden, C., Paulson, D.B., Asplund, M., BlandHawthorn, J., Bessell, M.S., and Freeman, K.C. (2006) Chemical homogeneity in the Hyades. Astron J 131:455-460.

Delgado Mena, E., Israelian, G., González Hernández, J.I., Bond, J.C., Santos, N.C., Udry, S., and Mayor, M. (2010) Chemical clues on the formation of planetary systems: $\mathrm{C} / \mathrm{O}$ versus $\mathrm{Mg} / \mathrm{Si}$ for HARPS GTO sample. Astrophys $J$ 725:2349-2358.

Desch, S.J. (2007) Mass distribution and planet formation in the solar nebula. Astrophys J 671:878-893.

Desch, S.J. (2012) Snow lines in external photoevaporated protoplanetary disks. [abstract 1659]. In 43 ${ }^{\text {rd }}$ Lunar and Planetary Science Conference Abstracts, Lunar and Planetary Science Institute, Houston.

Desch, S.J., Young, P.A., Anbar, A.D., Hinkel, N., Pagano, M., Truitt, A., and Turnbull, M. (2014) Report on a NASA Astrobiology Institute-Funded Workshop Without Walls: Stellar Stoichiometry. Astrobiology 14:271-276.

Desert, J.-M., Lecavelier Des Etangs, A., Hébrard, G., Sing, D.K., Ehrenreich, D., Ferlet, R., and Vidal-Madjar, A. (2009) Search for carbon monoxide in the atmosphere of the transiting exoplanet HD 189733b. Astrophys J 699:478-485.

Dressing, C. and Charbonneau, D. (2013) The occurrence rate of small planets around small stars. Astrophys $J$ 767:95-115.

Dye, S.T. (2012) Geoneutrinos and the radioactive power of the Earth. Rev Geophys 50:RG3007.

Ebel, D.S. (2000) Condensation in dust-enriched systems. Geochim Cosmochim Acta 64:339-366.
Ebel, D.S. (2013) Condensation of Rocky Material in Astrophysical Environments in Meteorites and the Early Solar System II, University of Arizona Press, Tucson.

Edvardsson, B., Andersen, J., Gustafsson, B., Lambert, D.L., Nissen, P.E., and Tomkin, J. (1993) The chemical evolution of the galactic disk. I. Analysis and results. Astron Astrophys 275:101-152.

Ellinger, C.I., Young, P.A., Fryer, C.L., and Rockefeller, G. (2012) A case study of small-scale structure formation in threedimensional supernova simulations. Astrophys J 755:160-194.

Fabbian, D., Asplund, M., Barklem, P.S., Carlsson, M., and Kiselman, D. (2009) Neutral oxygen spectral line formation revisited with new collisional data: large departures from LTE at low metallicity. Astron Astrophys 500:1221-1238.

Fedkin, A.V., Meyer, B.S., and Grossman, L. (2010) Condensation and mixing in supernova ejecta. Geochim Cosmochim Acta 74:3642-3658.

Ferraz-Mello, S., Rodríguez, A., and Hussmann, H. (2008) Tidal friction in close-in satellites and exoplanets: the Darwin theory re-visited. Celestial Mechanics and Dynamical Astronomy 101:171-201.

Fesen, R.A., Morse, J.A., Chevalier, R.A., Borkowski, K.J., Gerardy, C.L., Lawrence, S.S., and van der Bergh, S. (2001) Hubble Space Telescope WFPC2 imaging of Cassiopeia A. Astron J 122:2644-2661

Fortney, J.J. (2012) On the carbon-to-oxygen ratio measurement in nearby Sun-like stars: implications for planet formation and the determination of stellar abundances. Astrophys $J$ 747, doi:10.1088/2041-8205/747/2/L27.

Fortney, J.J., Marley, M.S., Lodders, K., Saumon, D., and Freedman, R. (2005) Comparative planetary atmospheres: models of TrES-1 and HD 209458b. Astrophys J Lett 627:L69-L72.

Fortney, J.J., Marley, M.S., and Barnes, J.W. (2007) Planetary radii across five orders of magnitude in mass and stellar insolation: application to transits. Astrophys J 659:1661-1672.

Foster, P.N. and Boss, A.P. (1995) Triggering star formation with stellar ejecta. Astrophys J 468:784-796.

Fryxell, B., Olson, K., Ricker, P., Timmes, F.X., Zingale, M., Lamb, D.Q., MacNeice, P., Rosner, R., Truran, J.W., and Tufo, H. (2000) FLASH: an adaptive mesh hydrodynamics code for modeling astrophysical thermonuclear flashes. Astrophys J Suppl Ser 131, doi:10.1086/317361.

Gillon, M., Demory, B.-O., Benneke, B., Valencia, D., Deming, D., Seager, S., Lovis, Ch., Mayor, M., Pepe, F., Queloz, D., Ségransan, D., and Udry, S. (2012) Improved precision on the radius of the nearby super-Earth $55 \mathrm{Cnc}$ e. Astron Astrophys 539, doi:10.1051/0004-6361/201118309.

González Hernández, J.I., Israelian, G., Santos, N.C., Sousa, S., Delgado-Mena, E., Neves, V., and Udry, S. (2010) Searching for the signatures of terrestrial planets in solar analogs. Astrophys $J$ 720:1592-1602.

Goriely, S., Bauswein, A., and Janka, H.-T. (2011) r-process nucleosynthesis in dynamically ejected matter of neutron star mergers. Astrophys J 738, doi:10.1088/2041-8205/738/2/L32.

Gounelle, M., Meibom, A., Hennebelle, P., and Inutsuka, S. (2009) Supernova propagation and cloud enrichment: a new model for the origin of ${ }^{60} \mathrm{Fe}$ in the early Solar System. Astrophys J 649:L1-L5.

Grocholski, B., Shim, S.-H., and Prakapenka, V.B. (2013) Stability, metastability, and elastic properties of a dense silica polymorph, seifertite. J Geophys Res 118:4745-4757.

Guillot, T. and Hueso, R. (2006) The composition of Jupiter: sign of a (relatively) late formation in a chemically evolved protosolar disc. Mon Not R Astron Soc 367:L47-L51. 
Gullbring, E., Hartmann, L., Briceno, C., and Calvet, N. (1998) Disk accretion rates for $\mathrm{T}$ Tauri stars. Astrophys J 492: 323-331.

Hansen, C., Kawaler, S., and Trimble, W. (2004) Stellar Interiors, Springer, New York.

Harder, H. and Schubert, G. (2001) Sulfur in Mercury's core? Icarus 151:118-122.

Hart, M.H. (1978) The evolution of the atmosphere of the Earth Icarus 33:23-39.

Hartmann, L., Calvet, N., Gullbring, E., and D'Alessio, P. (1998) Accretion and the evolution of T Tauri disks. Astrophys J 495:385-400.

Helfer, H.L., Wallerstein, G., and Greenstein, J.L. (1959) Abundances of some population II K giants. Astrophys J 129:700-720.

Hemley, R.J., editor. (1998) Ultrahigh-Pressure Mineralogy: Physics and Chemistry of the Earth's Deep Interior, Reviews in Mineralogy Vol. 37, Mineralogical Society of America, Washington, DC.

Hester, J.J. and Desch, S.J. (2005) Understanding our origins: star formation in H II region environments. In Chondrites and the Protoplanetary Disk, ASP Conference Series 341, edited by A.N. Krot, E.R.D. Scott, and B. Reipurth, Astronomical Society of the Pacific, San Francisco, pp 107-130.

Hinkel, N.R. (2012) Stellar abundances in the solar neighborhood. PhD thesis, Arizona State University, Tempe.

Hinkel, N.R. and Kane, S.R. (2013) Implications of the spectroscopic abundances in $\alpha$ Centauri A and B. Mon Not $R$ Astron Soc 432:L36.

Hirose, K., Takafuji, N., Sata, N., and Ohishi, Y. (2005) Phase transition and density of subducted MORB crust in the lower mantle. Earth Planet Sci Lett 237:239-251.

Holweger, H. (1979) Abundances of the elements in the Sun. Introductory report in The Elements and Their Isotopes in the Universe; International Conference on Astrophysics, Universite de Liege, Cointe-Ougree, Belgium, pp 117-138.

Huang, S.S. (1959) Occurrence of life in the Universe. Am Sci 47:397-402.

Hurford, T.A., Helfenstein, P., Hoppa, G.V., Greenberg, R., and Bills, B.G. (2007) Eruptions arising from tidally controlled periodic openings of rifts on Enceladus. Nature 447:292-294.

Huss, G.R., Meyer, B.S., Srinivasan, G., Goswami, J.N., and Sahijpal, S. (2009) Stellar sources of the short-lived radionuclides in the early Solar System. Geochim Cosmochim Acta 73:4922-4945.

Hut, P. (1981) Tidal evolution in close binary systems. Astron Astrophys 99:126-140.

Hwang, U., Laming, J.M., Badenes, C., Berendse, F., Blondin, J., Cioffi, D., DeLaney, T., Dewey, D., Fesen, R., Flanagan, K.A., Fryer, C.L., Ghavamian, P., Hughes, J.P., Morse, J.A., Plucinsky, P.P., Petre, R., Pohl, M., Rudnick, L., Sankrit, R., Slane, P.O., Smith, R.K., Vink, J., and Warren, J.S. (2004) A million second Chandra view of Cassopeia A. Astrophys $J$ 615:L117-L120.

Iglesias, C.A. and Rogers, F.J. (1996) Updated OPAL opacities. Astrophys J 464:943-953.

Irwin, P.G.J., Weir, A.L., Smith, S.E., Taylor, F.W., Lambert, A.L., Calcutt, S.B., Cameron-Smith, P.J., Carlson, R.W., Baines, K., Orton, G.S., Drossart, P., Encrenaz, T., and RoosSerote, M. (1998) Cloud structure and atmospheric composition of Jupiter retrieved from Galileo near-infrared mapping spectrometer real-time spectra. J Geophys Res 103:2300123022.
Isobe, T., Feigelson, E.D., Akritas, M.G., and Babu, G.J. (1990) Linear regression in astronomy. Astrophys J 364:104-113.

Ito, E. and Takahashi, E. (1989) Postspinel transformations in the system $\mathrm{Mg}_{2} \mathrm{SiO}_{4}-\mathrm{Fe}_{2} \mathrm{SiO}_{4}$ and some geophysical implications. J Geophys Res 94:10637-10646.

Jackson, B., Barnes, R., and Greenberg, R. (2008) Tidal heating of terrestrial extrasolar planets and implications for their habitability. Mon Not R Astron Soc 391:237-245.

Kaltenegger, L., Sasselov, D., and Rugheimer, S. (2013) Waterplanets in the habitable zone: atmospheric chemistry, observable features, and the case of Kepler-62e and -62f. Astrophys $J$ 775, doi:10.1088/2041-8205/775/2/L47.

KamLAND Collaboration. (2011) Partial radiogenic heat model for Earth revealed by geoneutrino measurements. Nat Geosci 4:647-651.

Kasting, J.F., Whitmire, D.P., and Reynolds, R.T. (1993) Habitable zones around main sequence stars. Icarus 101:108-128.

Kifonidis, K., Plewa, T., Janka, H.-Th., and Muller, E. (2003) Non-spherical core collapse supernovae. I. Neutrino-driven convection, Rayleigh-Taylor instabilities, and the formation and propagation of metal clumps. Astron Astrophys 408: 621-649.

Kopparapu, R., Kasting, J.F., and Zahnle, K.J. (2012) A photochemical model for the carbon-rich planet WASP-12b. Astrophys $J$ 745, doi:10.1088/0004-637X/745/1/77.

Kopparapu, R.K., Ramirez, R., Kasting, J.F., Eymet, V., Robinson, T.D., Mahadevan, S., Terrien, R.C., Domagal-Goldman, S., Meadows, V., and Deshpande, R. (2013) Habitable zones around main-sequence stars: new estimates. Astrophys J 765:131-147.

Kuchner, M. and Seager, S. (2005) Extrasolar carbon planets. arXiv:astro-ph/0504214.

Lambeck, K. (1977) Tidal dissipation in the oceans: astronomical, geophysical and oceanographic consequences. $\underline{P h i-}$ los Transact A Math Phys Eng Sci 287:545-594.

Larimer, J.W. (1975) The effect of C/O ratio on the condensation of planetary material. Geochim Cosmochim Acta 39:389-392.

Lecar, M., Podolak, M., Sasselov, D., and Chiang, E. (2006) On the location of the snow line in a protoplanetary disk. Astrophys J 640:1115-1118.

Leconte, J., Chabrier, G., Baraffe, I., and Levrard, B. (2010) Is tidal heating sufficient to explain bloated exoplanets? Consistent calculations accounting for finite initial eccentricity. Astron Astrophys 516:A64.

Lesniak, M.V. and Desch, S.J. (2011) Temperature structure of protoplanetary disks undergoing layered accretion. Astrophys J 740:118-136.

Lodders, K. (2003) Solar system abundances and condensation temperatures of the elements. Astrophys J 591:1220-1247.

Lodders, K. (2004) Jupiter formed with more tar than ice. Astrophys J 611:587-597.

Lodders, K. (2010) Solar System abundances of the elements. In Principles and Perspectives in Cosmochemistry: Lecture Notes of the Kodai School on "Synthesis of Elements in Stars" Held at Kodaikanal Observatory, India, April 29-May 13, 2008, edited by A. Goswami and B.E. Reddy, SpringerVerlag, Berlin, Heidelberg, pp 378-417.

Lodders, K. and Fegley, B. (2002) Atmospheric chemistry in giant planets, brown dwarfs, and low-mass dwarf stars. I. Carbon, nitrogen, and oxygen. Icarus 155:393-424.

Ludhova, L. (2012) Geo-neutrinos. Geoscientific Instrumentation, Methods and Data Systems 1:221-227.

Lynden-Bell, D. and Pringle, J.E. (1974) The evolution of viscous discs and the origin of the nebular variables. Mon Not $R$ Astron Soc 168:603-637. 
Madhusudhan, N. (2012) C/O ratio as a dimension for characterizing exoplanetary atmospheres. Astrophys $J$ 758, doi:10.1088/0004-637X/758/1/36.

Madhusudhan, N. and Seager, S. (2009) A temperature and abundance retrieval method for exoplanet atmospheres. Astrophys J 707:24-39.

Madhusudhan, N., Harrington, J., Stevenson, K.B., Nymeyer, S., Campo, C.J., Wheatley, P.J., Deming, D., Blecic, J., Hardy, R.A., Lust, N.B., Anderson, D.R., Collier-Cameron, A., Britt, C.B., Bowman, W.C., Hebb, L., Hellier, C., Maxted, P.F., Pollacco, D., and West, R.G. (2011a) A high C/O ratio and weak thermal inversion in the atmosphere of exoplanet WASP-12b. Nature 469:64-67.

Madhusudhan, N., Mousis, O., Johnson, T.V., and Lunine, J.I. (2011b) Carbon-rich giant planets: atmospheric chemistry, thermal inversions, spectra, and formation conditions. Astrophys $J$ 743, doi:10.1088/0004-637X/743/2/191.

Madhusudhan, N., Lee, K.K.M., and Mousis, O. (2012) A possible carbon-rich interior in super-Earth 55 Cancri e. Astrophys $J$ 759, doi:10.1088/2041-8205/759/2/L40.

Mahaffy, P.R., Niemann, H.B., Alpert, A., Atreya, S.K., Demick, J., Donahue, T.M., Harpold, D.N., and Owen, T.C. (2000) Noble gas abundance and isotope ratios in the atmosphere of Jupiter from the Galileo probe mass spectrometer. $\underline{J}$ Geophys Res 105:15061-15072.

Matousek, S. (2007) The Juno New Frontiers mission. Acta Astronaut 61:932-939.

McDonough, W.F. and Sun, S.-S. (1995) The composition of the Earth. Chem Geol 120:223-253.

Mishenina, T.V., Soubiran, C., Bienaymè, O., Korotin, S.A., Belik, S.I., Usenko, I.A., and Kovtyukh, V.V. (2008) Spectroscopic investigation of stars on the lower main sequence. Astron Astrophys 489:923-930.

Morbidelli, A., Brown, M.E., and Levison, H.F. (2003) The Kuiper Belt and its primordial sculpting. Earth Moon Planets 92:1-27.

Moses, J.L., Line, M.R., Visscher, C., Richardson, M.R., Nettelmann, N., Fortney, J.J., Barman, T.S., Stevenson, K.B., and Madhusudhan, N. (2013) Compositional diversity in the atmospheres of hot Neptunes, with application to GJ 436b. Astrophys $J$ 777:34-57.

Mousis, O., Lunine, J.I., Madhusudhan, N., and Johnson, T.V. (2012) Nebular water depletion as the cause of Jupiter's low oxygen abundance. Astrophys J 751, doi:10.1088/2041-8205/ 751/1/L7.

Neves, V., Santos, N.C., Sousa, S.G., Correia, A.C.M., and Israelian, G. (2009) Chemical abundances of 451 stars from the HARPS GTO planet search program. Thin disc, thick disc, and planets. Astron Astrophys 497:563-581.

Niemann, H.B., Atreya, S.K., Carignan, G.R., Donahue, T.M., Haberman, J.A., Harpold, D.N., Hartle, R.E., Hunten, D.M., Kasprzak, W.T., Mahaffy, P.R., Owen, T.C., and Way, S.H. (1998) The composition of the jovian atmosphere as determined by the Galileo probe mass spectrometer. J Geophys Res 103:22831-22846.

Nissen, P.E. (2013) The carbon to oxygen ratio in stars with planets. Astron Astrophys 552:73-83.

Öberg, K., Murray-Clay, R., and Bergin, E.A. (2011) The effects of snowlines on $\mathrm{C} / \mathrm{O}$ in planetary atmospheres. Astrophys $J$ 743, doi:10.1088/2041-8205/743/1/L16.

Ouellette, N., Desch, S.J., and Hester, J.J. (2007) Interaction of supernova ejecta with nearby protoplanetary disks. Astrophys J 662:1268-1281.
Ouellette, N., Desch, S.J., and Hester, J.J. (2010) Injection of supernova dust in nearby protoplanetary disks. Astrophys $J$ 711:597-612.

Owen, T., Bar-Nun, A., and Kleinfeld, I. (1992) Possible cometary origin of heavy noble gases in the atmospheres of Venus, Earth, and Mars. Nature 358:43-46.

Pan, L., Desch, S.J., Scannapieco, E., and Timmes, F.X. (2012) Mixing of clumpy supernova ejecta into molecular clouds. Astrophys J 756:102-122.

Peale, S.J., Cassen, P., and Reynolds, R.T. (1979) Melting of Io by tidal dissipation. Science 203:892-894.

Petigura, E.A. and Marcy, G.W. (2011) Carbon and oxygen in nearby stars: keys to protoplanetary disk chemistry. Astrophys $J$ 735, doi: 10.1088/0004-637X/735/1/41.

Queloz, D., Bouchy, F., Moutou, C., Hatzes, A., Hébrard, G., Alonso, R., Auvergne, M., Baglin, A., Barbieri, M., Barge, P., Benz, W., Bordé, P., Deeg, H.J., Deleuil, M., Dvorak, R., Erikson, A., Ferraz Mello, S., Fridlund, M., Gandolfi, D., Gillon, M., Guenther, E., Guillot, T., Jorda, L., Hartmann, M., Lammer, H., Léger, A., Llebaria, A., Lovis, C., Magain, P., Mayor, M., Mazeh, T., Ollivier, M., Pätzold, M., Pepe, F., Rauer, H., Rouan, D., Schneider, J., Segransan, D., Udry, S., and Wuchterl, G. (2009) The CoRoT-7 planetary system: two orbiting super-Earths. Astron Astrophys 506:309-319.

Ramírez, I., Allende Prieto, C., and Lambert, D.L. (2007) Oxygen abundances in nearby stars. Clues to the formation and evolution of the galactic disk. Astron Astrophys 465:271-281.

Ramírez, I., Meléndez, J., and Asplund, M. (2009) Accurate abundance patterns of solar twins and analogs. Does the anomalous solar chemical composition come from planet formation? Astron Astrophys 508:L17-L20.

Raymond, S.N., Scalo, J., and Meadows, V.S. (2007) A decreased probability of habitable planet formation around lowmass stars. Astrophys J 669:606-614.

Ricolleau, A., Fiquet, G., Addad, A., Menguy, N., Vanni, C., Perrillat, J.-P., Daniel, I., Cardon, H., and Guignot, N. (2008) Analytical transmission microscopy study of a natural MORB sample assemblage transformed at high pressure and high temperature. Am Mineral 93:144-153.

Riner, M.A., Bina, C.R., Robinson, M.S., and Desch, S.J. (2008) Internal structure of Mercury: implications of a molten core. J Geophys Res 113, doi:10.1029/2007JE002993.

Ringwood, A.E. (1970) Phase transformations and the constitution of the mantle. Physics of the Earth and Planetary Interiors 3:109-155.

Ringwood A.E. (1975) Composition and Petrology of the Earth's Mantle, McGraw-Hill, New York.

Ringwood, A.E. (1977) Composition of the core and implications for origin of the Earth. Geochem J 11:111-135.

Rogers, L.A. and Seager, S. (2010) A framework for quantifying the degeneracies of exoplanet interior compositions. Astrophys J 712:974-991.

Rogers, F.J., Swenson Rogers, F.J., and Iglesias, C.A. (1996) OPAL equation-of-state tables for astrophysical applications. Astrophys J 456:902-908.

Schönrich, R. and Binney, J. (2009) Chemical evolution with radial mixing. Mon Not R Astron Soc 396:203-222.

Seager, S., Richardson, L.J., Hansen, B.M.S., Menou, K., Cho, J.Y.-K., and Deming, D. (2005) On the dayside thermal emission of hot Jupiters. Astrophys J 632:1122-1131.

Seager, S., Kuchner, M., Hier-Majumder, C.A., and Militzer, B. (2007) Mass-radius relationships for solid exoplanets. Astrophys J 669:1279-1297. 
Selsis, F., Kasting, J.F., Levrard, B., Paillet, J., Ribas, I., and Delfosse, X. (2007) Habitable planets around the star Gliese 581? Astrophys J 476:1373-1387.

Sen, S., Widgeon, S.J., Navrotsky, A., Mera, G., Tavakoli, A., Ionescu, E., and Riedel, R. (2013). Carbon substitution for oxygen in silicates in planetary interiors. Proc Natl Acad Sci USA 110:15904-15907.

Shim, S.-H., Duffy, T.S., and Shen, G. (2001) The post-spinel transformation in $\mathrm{Mg}_{2} \mathrm{SiO}_{4}$ and its relation to the $660-\mathrm{km}$ seismic discontinuity. Nature 411:571-574.

Snider, K.D., Hester, J.J., Desch, S.J., Healy, K.R., and Bally, J. (2009) Spitzer observations of the H II region NGC 2467: an analysis of triggered star formation. Astrophys J 700:506-522.

Steigman, G. (2012) Neutrinos and Big Bang nucleosynthesis. arXiv.1208.0032

Stevenson, D.J. and Lunine, J.I. (1988) Rapid formation of Jupiter by diffuse redistribution of water vapor in the solar nebula. Icarus 75:146-155.

Stevenson, D.J. and Salpeter, E.E. (1977a) The phase diagram and transport properties for hydrogen-helium fluid planets. Astrophys J Suppl Ser 35:221-238.

Stevenson, D.J. and Salpeter, E.E. (1977b) The dynamics and helium distribution in hydrogen-helium fluid planets. Astrophys J Suppl Ser 35:239-261.

Stishov, S.M. and Popova, S.V. (1961) New dense polymorphic modification of silica. Geokhimiya 10:837-839.

Stuart, A. and Ord, K. (2009) Kendall's Advanced Theory of Statistics, Volume 1: Distribution Theory, Wiley, New York.

Swain, M.R., Vasisht, G., and Tinetti, G. (2009) Molecular signatures in the near-infrared dayside spectrum of HD 189733b. Astrophys J 690:L114-L117.

Takeda, Y. (2003) Oxygen line formation in late-F through early-K disk/halo stars. Infrared $\mathrm{O}_{\mathrm{I}}$ triplet and [O I] lines. Astron Astrophys 402:343-359.

Takeda, Y. (2007) Fundamental parameters and elemental abundances of 160 F-G-K stars based on OAO spectrum database. Publications of the Astronomical Society of Japan 59:335-356.

Taylor, S.R. and McLennan, S.M. (1985) The Continental Crust: Its Composition and Evolution: An Examination of the Geochemical Record Preserved in Sedimentary Rocks, Blackwell Scientific, Oxford.

Teske, J.K., Cunha, K., Schuler, S.C., Griffith, C.A., and Smith, V.V. (2013) Carbon and oxygen abundances in cool metalrich exoplanet hosts: a case study of the $\mathrm{C} / \mathrm{O}$ ratio of 55 Cancri. Astrophys J 778:132-141.

Thévenin, F. and Idiart, T.P. (1999) Stellar iron abundances: non-LTE effects. Astrophys J 521:753-763.

Timmes, F.X., Woosley, S.E., and Weaver, T.A. (1995) Galactic chemical evolution: hydrogen through zinc. Astrophys $J$ Suppl Ser 98:617-658.

Tinsley, B.M. (1980) Evolution of the stars and gas in galaxies. Fundamentals of Cosmic Physics 5:287-388.

Tolich, N., for the SNO Collaboration. (2012) Final results from SNO. Journal of Physics: Conference Series 375, doi:10.1088/ 1742-6596/375/1/042049.

Urey, H.C. (1951) The origin and development of the Earth and other terrestrial planets. Geochim Cosmochim Acta 1:209-277.

Valencia, D., O'Connell, R.J., and Sasselov, D.D. (2007) Inevitability of plate tectonics on super-Earths. Astrophys $J$ 670:L45-L48.

Valencia, D., Ikoma, M., Guillot, T., and Nettelmann, N. (2010) Composition and fate of short-period super-Earths. The case of CoRoT-7b. Astron Astrophys 516, doi:10.1051/0004-6361/ 200912839.

Valenti, J.A. and Fischer, D.A. (2005) Spectroscopic Properties of Cool Stars (SPOCS). I. 1040 F, G, and K Dwarfs from Keck, Lick, and AAT Planet Search Programs. Astrophys J Suppl Ser 159:141-166.

Wadhwa, M., Amelin, Y., Davis, A.M., Lugmair, G.W., Meyer, B., Gounelle, M., and Desch, S.J. (2007) From dust to planetesimals: implications for the solar protoplanetary disk from short-lived radionuclides. In Protostars and Planets $V$, edited by B. Reipurth, D. Jewitt, and K. Keil, University of Arizona Press, Tucson, pp 835-848.

Walker, J.C.G., Hays, P.B., and Kasting, J.F. (1981) A negative feedback mechanism for the long-term stabilization of Earth's surface climate. J Geophys Res 86:9776-9782.

Wilson, H.F. and Militzer, B. (2010) Sequestration of noble gases in giant planet interiors. Phys Rev Lett 104, doi:10.1103/ PhysRevLett.104.121101.

Winn, J.N., Matthews, J.M., and Dawson, R.I. (2011) A superEarth transiting a naked-eye star. Astrophys J 737, doi:10.1088/ 2041-8205/737/1/L18.

Wong, M.H., Mahaffy, P.R., Atreya, S.K., Niemann, H.B., and Owen, T.C. (2004) Updated Galileo probe mass spectrometer measurements of carbon, oxygen, nitrogen, and sulfur on Jupiter. Icarus 171:153-170.

Wordsworth, R.D., Forget, F., Selsis, F., Millour, E., Charnay, B., and Madeleine, J.-B. (2011) Gliese 581d is the first discovered terrestrial-mass exoplanet in the habitable zone. Astrophys $J$ 733, doi:10.1088/2041-8205/733/2/L48.

Yang, J., Cowan, N.B., and Abbot, D.S. (2013) Stabilizing cloud feedback dramatically expands the habitable zone of tidally locked planets. Astrophys J 771, doi:10.1088/20418205/771/2/L45.

Yoshida, M., Onodera, A., Ueno, M., Takemura, K., and Shimomura, O. (1993) Pressure-induced phase transition in SiC. Phys Rev B 48:10587-10590.

Young, E.D., Gounelle, M., Smith, R., Morris, M.R., and Pontoppidan, K.M. (2011) Astronomical oxygen isotopic evidence for supernova enrichment of the Solar System birth environment. Astrophys J 729:43-55.

Young, P.A. and Arnett, D. (2005) Observational tests and predictive stellar evolution. II. Nonstandard models. Astrophys $J$ 618:908-918.

Young, P.A., Ellinger, C.I., Arnett, D., Fryer, C.L., and Rockefeller, G. (2009) Finding tracers for supernova produced ${ }^{26}$ Al. Astrophys J 699, doi:10.1088/0004-637X/699/2/938.

Young, P.A., Liebst, K., and Pagano, M. (2012) The impact of stellar abundance variations on stellar habitable zone evolution. Astrophys J 755, doi:10.1088/2041-8205/755/2/L31.

Zindler, A. and Hart, S. (1986) Chemical geodynamics. Annu Rev Earth Planet Sci 14:493-571.

Address correspondence to: Patrick Young School of Earth and Space Exploration Arizona State University PO Box 871404 Tempe AZ, 85287-1404

E-mail: Patrick.young.1@asu.edu

Submitted 14 January 2014 Accepted 4 April 2014 
This article has been cited by:

1. Desch Steven J., Young Patrick A., Anbar Ariel D., Hinkel Natalie, Pagano Michael, Truitt Amanda, Turnbull Margaret. 2014. Report on a NASA Astrobiology Institute-Funded Workshop Without Walls: Stellar Stoichiometry. Astrobiology 14:4, 271-276. [Abstract] [Full Text HTML] [Full Text PDF] [Full Text PDF with Links] 